\title{
In vivo enhancement of anticancer therapy using bare or chemotherapeutic drug-bearing nanodiamond particles
}

This article was published in the following Dove Press journal:

International Journal of Nanomedicine

2I February 20I4

Number of times this article has been viewed

\section{Yingqi Li, ${ }^{1,2}$ \\ Yaoli Tong' \\ Ruixia Cao' \\ Zhimei Tian ${ }^{2}$ \\ Binsheng Yang ${ }^{2}$ \\ Pin Yang ${ }^{2}$}

'Department of Chemistry, College of Chemistry and Chemical Engineering, ${ }^{2}$ Key Laboratory of Chemical Biology and Molecular Engineering of Ministry of Education, Institute of Molecular Science, Shanxi University, Taiyuan, People's Republic of China

\section{Video abstract}

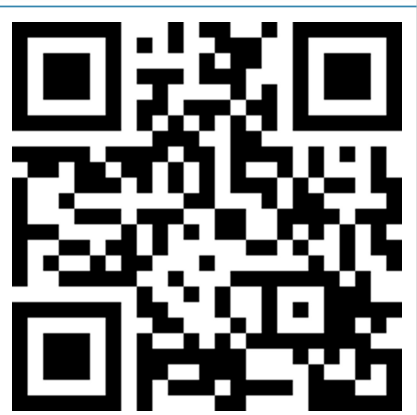

Point your SmartPhone at the code above. If you have a QR code reader the video abstract will appear. Or use: http://dvpr.es//hosTxK

Correspondence: Yingqi Li; Binsheng Yang Key Laboratory of Chemical Biology and Molecular Engineering of Ministry of Education, Institute of Molecular Science, Shanxi University, Taiyuan 030006,

People's Republic of China

Tel +8635 I 70 I 0588

Email wkyqli@sxu.edu.cn; yangbs@sxu. edu
Background: This study investigated the use of nanodiamond particles (NDs) as a promising material for drug delivery in vivo and in vitro.

Methods: HepG2 cells (a human hepatic carcinoma cell line) were used to determine the characteristics of a nanodiamond-doxorubicin complex (ND-DOX) when taken up by cells in vitro using laser scanning confocal microscopy and dialysis experiments. We also compared the survival rate and histopathology of tumor-bearing mice after treatment with NDs or NDDOX in vivo.

Results: In vitro investigation showed that ND-DOX has slow and sustained drug release characteristics compared with free doxorubicin. In vivo, the survival rate of tumor-bearing mice treated with ND-DOX was four times greater than that of mice treated with free doxorubicin. Interestingly, the survival rate in mice treated with NDs alone was close to that of mice treated with free doxorubicin. This indicates that treatment with ND-DOX can prolong the lifespan of tumor-bearing mice significantly compared with conventional doxorubicin and that NDs can have this effect as well. Histopathological analysis showed that neither the NDs nor ND-DOX were toxic to the kidney, liver, or spleen in contrast with the well-known toxic effects of free doxorubicin on the kidney and liver. Further, both the bare NDs and ND-DOX could suppress tumor growth effectively.

Conclusion: NDs can potentially prolong survival, and ND-DOX may act as a nanodrug with promising chemotherapeutic efficacy and safety.

Keywords: nanodiamond, drug delivery, sustained release, survival rate, cancer, treatment

\section{Introduction}

In recent years, carbon-based nanomaterials, such as fullerenes and nanotubes, have gained widespread attention for biological application due to their physical, chemical, and biological properties. ${ }^{1-6}$ However, the biocompatibility of these nanomaterials remains in question, hence limiting their use in the clinical setting. There is significant interest in nanodiamond particles (NDs) owing to their low toxicity, high chemical stability, high affinity for biomolecules, and ease of surface functionalization. ${ }^{7-9} \mathrm{Com}-$ paring carbon-based nanomaterials in vitro, Schrand et al showed that ND had the greatest biocompatibility, followed sequentially by carbon black, multiwalled carbon nanotubes, and single-walled carbon nanotubes. ${ }^{10}$ Similarly, Zhang et al concluded that NDs showed the highest cell uptake rate and the least cytotoxicity when compared with multiwalled carbon nanotubes and graphene oxide. ${ }^{11}$ In the past few years, there have been several studies on the biocompatibility and biodistribution of NDs in vivo. For example, Puzyr et al ${ }^{12}$ demonstrated the absence of an obvious inflammatory response 
at 3 months post-dosing in mice injected subcutaneously with modified NDs. However, a number of biochemical indices in rabbit blood were affected after intravenous administration of modified NDs. NDs with a diameter of around $50 \mathrm{~nm}$ were found by radiolabeling to accumulate predominantly in the liver after intravenous injection in mice. ${ }^{13}$

One of the most noteworthy applications of NDs is as red fluorescence nanodiamond (FND). FND can serve as a biomedical imaging agent because its diamond core shows no signs of photobleaching even after its surface is functionalized with various biomolecules. ${ }^{14-18}$ Mohan et al used bare FND or FND conjugated with biomolecules for in vivo imaging of Caenorhabditis elegans ${ }^{19}$ and found no detectable changes in reactive oxygen species. Recently, Rojas et $\mathrm{al}^{20}$ studied the in vivo biodistribution of detonation nanodiamond labeled with ${ }^{18} \mathrm{~F}$ in rats using positron emission tomography. More recently, Vaijayanthimala et $\mathrm{al}^{21}$ investigated the long-term stability and biocompatibility of $100 \mathrm{~nm}$ FND administered by intraperitoneal injection over 5 months in rats.

Application of nanotechnology for drug delivery in the treatment of cancer is presently a topic of intense interest. ${ }^{22-24}$ Despite numerous studies of NDs, their use as a drug delivery system needs to be evaluated further. A critical challenge is the ability to deliver a sufficient amount of drug to the desired location with fewer side effects than those associated with conventional therapies. Doxorubicin is an effective and widely used chemotherapeutic agent. However, its clinical application is limited by its dose-related toxicity, including cumulative cardiotoxicity and myelosuppression. Recently, Huang et $\mathrm{al}^{25}$ and Chow et $\mathrm{al}^{26}$ absorbed doxorubicin hydrochloride onto NDs (2-8 $\mathrm{nm}$ or $45 \mathrm{~nm}$ in size) and investigated their activity in cells and in mouse models of liver and mammary cancer. They demonstrated that NDs can be used as drug delivery vehicles, with a complex of nanodiamond and doxorubicin (ND-DOX) overcoming chemoresistance and enhancing chemotherapeutic efficacy and safety. However, the in vivo effects of administering pristine NDs or ND-DOX with particle sizes greater than $100 \mathrm{~nm}$ to treat tumor-bearing mice are unknown, and no relevant information on this topic has been published as yet.

It is well known that particles $100-400 \mathrm{~nm}$ in diameter ${ }^{27}$ tend to accumulate in tumor tissues more readily than in normal tissues because newly formed blood vessels within a tumor are usually abnormal in form and architecture, poorly aligned, have wide fenestrations, and lack a smooth muscle layer. Further, tumors generally lack effective lymphatic drainage. ${ }^{28,29}$ In view of these observations, we were interested to know, for example, what are the function and fate of bare
NDs (mean $140 \mathrm{~nm}$ ) in vivo with a complex system. What is the difference in the complex of ND-DOX (mean 165 $\mathrm{nm}$ ) compared with doxorubicin alone in vitro or in vivo? With these questions in mind, we carried out these materials mentioned above in vitro and in vivo efficacy.

Here we report the results of our study on the function and fate of bare NDs (mean diameter $140 \mathrm{~nm}$ ) and an NDDOX complex (mean diameter $165 \mathrm{~nm}$ ) administered by intraperitoneal injection in a tumor-bearing mouse model. This work is an extension of our previous studies of ND-DOX in vitro, ${ }^{30}$ in which we confirmed that ND-DOX has anticancer efficacy in vitro and shows slow and sustained drug release capability. It is important to determine if NDs can deliver a chemotherapeutic agent to solid tumors in animals via the enhanced permeability and retention effect. Here, the ND-DOX complex showed sustained release characteristics in vitro. Interestingly, bare NDs were able to suppress tumor growth in vivo. The ND-DOX delivery system developed in this study improved treatment safety and efficacy in tumorbearing mice when compared with doxorubicin alone, suggesting that NDs are a promising targeting delivery platform for chemotherapeutic drugs. Further studies of bare and drug-loaded NDs will have far reaching significance.

\section{Materials and methods Materials and measurements}

Synthetic type Ib NDs with a diameter of $140 \mathrm{~nm}$ were chosen for our experiments because blood vessels in tumor tissue can retain particles $100-400 \mathrm{~nm}$ in size. They were obtained from Element Six (Shannon, Ireland) FND was donated by the Institute of Atomic and Molecular Sciences, Academia Sinica, Taiwan. Doxorubicin hydrochloride was purchased from Shenzhen Main Luck Pharmaceuticals Inc (Shenzhen, People's Republic of China). HepG2 cells were provided by the Gene Engineering Center of Shanxi University (Taiyuan, People's Republic of China). Dulbecco's Modified Eagle's Medium (DMEM) was purchased from Thermo Fisher Biological and Chemical Products (Beijing, People's Republic of China). Fetal bovine serum was sourced from Hangzhou Sijiqing Biological Engineering Materials Co, Ltd (Hangzhou, People's Republic of China). Trypsin was purchased from the Sino-American Biotechnology Company (Zhengzhou, People's Republic of China). Ethylenediamine tetraacetic acid and 4\% paraformaldehyde were obtained from Beijing Solarbio Science and Technology Co, Ltd (Beijing, People's Republic of China). BisBenzimide (Hoechst 33258 , blue fluorescent dye) for staining cell nuclei was purchased from Beyotime Institute of Biotechnology (Jiangsu, 
People's Republic of China). Filtered water (EMD Millipore Corporation, Billerica, MA, USA) was used for all aqueous solutions. The list of equipment and instruments used is as follows: water-jacketed $\mathrm{CO}_{2}$ cell incubator (Shanghai Lishen Scientific Instruments Co, Ltd, Shanghai, People's Republic of China), FQ-100DE numerical control ultrasonic wave washing machine (Kunshan Ultrasonic Instrument Co, Ltd, Kunshan City, People's Republic of China) laser scanning confocal microscope (TCS SP5, Leica, Mannheim, Germany), fully automated microplate reader (Model 550, Bio-Rad Laboratories Inc, Hercules, CA, USA), fluorescence microscope (IX71, Olympus, Tokyo, Japan), flow cytometer (FACSCalibur' ${ }^{\mathrm{TM}}$, BD Biosciences, Franklin Lakes, NJ, USA), Zetasizer Nano ZS90 (Malvern Instruments Ltd, Malvern, UK) and FTIR-8400S infrared spectrophotometer (Shimadzu Corporation, Kyoto, Japan).

\section{Preparation of ND-DOX}

Factors affecting absorption of doxorubicin onto the NDs were investigated with $\mathrm{pH}$ adjusted in the range of 3.0-10.0 by hydrochloric acid and sodium hydroxide. On the basis of our results, we freshly prepared ND-DOX in sodium hydroxide solution at $\mathrm{pH} 10.0$ for maximum absorption of doxorubicin (the $\mathrm{pH}$ of the final solution was 7.56). Briefly, $2 \mathrm{mg}$ of NDs were sonicated at $100 \mathrm{~W}$ for 30-40 minutes in sodium hydroxide solution ( $\mathrm{pH} 10.0$ ). Next, $200 \mu \mathrm{g}$ of doxorubicin was added, and the suspension was shaken at room temperature for 3 hours to promote binding between doxorubicin and the NDs via physical adsorption. The suspension was then centrifuged at 15,000 rpm for 5 minutes, and the precipitate was washed three times with $\mathrm{pH} 10.0$ sodium hydroxide solution to remove the unadsorbed doxorubicin. The amount of doxorubicin adsorbed was determined by calculating the change in doxorubicin concentration before and after adsorption using an ultraviolet-visible spectrophotometer at $480 \mathrm{~nm}$. Particle size and zeta potential measurements were performed in distilled water using a Zetasizer Nano ZS90. Measurements of nanoparticle size were performed at $25^{\circ} \mathrm{C}$ and a scattering angle of $90^{\circ}$. The mean hydrodynamic diameter was determined by cumulative analysis. Determination of the zeta potential was based on electrophoretic mobility of the nanoparticles in aqueous medium, and was performed using folded capillary cells in automatic mode. IR spectra were carried out with a ShimadzuFTIR-8400S infrared spectrophotometer using $\mathrm{KBr}$ pelle.

\section{In vitro drug release study}

A tightly sealed dialysis bag containing a $2 \mathrm{~mL}$ suspension of ND-DOX or doxorubicin was placed into a $50 \mathrm{~mL}$ beaker and dialyzed in $0.01 \mathrm{M}$ phosphate-buffered saline $(9 \mathrm{~mL}$, $\mathrm{pH} 7.4,6.5$, and 5.5). At predetermined time intervals, the phosphate-buffered saline was removed and replaced with fresh medium. Dialysates were analyzed by ultraviolet-visible $(480 \mathrm{~nm})$ spectrophotometry to determine the concentration of dissociated doxorubicin and the cumulative release rate.

\section{Cell culture}

HepG2 cells are adherent, epithelial-like cells that grow as monolayers and in small aggregates, and are easy to obtain, so were chosen as the in vitro cell model for our experiments. The cells were cultured in a $10 \mathrm{~cm}$ Petri dish with glucose DMEM supplemented with $10 \%$ fetal bovine serum at $37^{\circ} \mathrm{C}$ in a humidified atmosphere containing $5 \% \mathrm{CO}_{2}$.

\section{Confocal microscopy}

We used laser confocal scanning microscopy to study the controlled drug release mechanism for ND-DOX, localization of ND-DOX, and release of doxorubicin within the intracellular space. Free doxorubicin was used as the control. Images were obtained using an inverted Leica confocal laser scanning microscope with FND-DOX instead of ND-DOX. The cells were seeded at a density of $2.0 \times 10^{5}$ cells onto a $35 \mathrm{~mm}$ culture dish with cover glass on the bottom.

Briefly, HepG2 cells were grown at $37^{\circ} \mathrm{C}$ in $5 \% \mathrm{CO}_{2}$. After 16 hours, the growth medium was removed, and cells were treated with either free doxorubicin $2 \mu \mathrm{g} / \mathrm{mL}$ for one hour or FND-DOX (FND $30 \mu \mathrm{g} / \mathrm{mL}+$ doxorubicin $2 \mu \mathrm{g} / \mathrm{mL}$ ) for one, 3,5 , and 16 hours. The free doxorubicin and FND-DOX were removed by washing the cells three times with phosphate-buffered saline. The cells were then fixed with $300 \mu \mathrm{L}$ of $4 \%$ paraformaldehyde for 8 minutes at room temperature. The nuclei were stained with $300 \mu \mathrm{L}$ of H33258. All images were obtained using an inverted Leica confocal laser scanning microscope. Samples stained with H33258 and FND were visualized at excitation wavelengths of $405 \mathrm{~nm}$ and $543 \mathrm{~nm}$, respectively. Sample fluorescence for the doxorubicin images was taken by excitation at $488 \mathrm{~nm}$. Collection of emission was from $630 \mathrm{~nm}$ to $750 \mathrm{~nm}$ for FND and from $530 \mathrm{~nm}$ to $580 \mathrm{~nm}$ for doxorubicin. In addition, all the FND-DOX nanoparticles used were sonicated for 30 minutes to thoroughly disperse them before use.

\section{Animals}

All the animal experiments were performed following the protocols approved by the Shanxi Tumor Institute (Taiyuan, People's Republic of China) using male Kun Ming mice aged 8-9 weeks and about $20 \mathrm{~g}$ in weight. The mice were supplied 
by the Animal Laboratory at the Shanxi Tumor Institute (production license SCXK [Jin] 2012-0001).

\section{Establishing the tumor-bearing mouse model}

Liver cancer (H22) cells were harvested from the mice when they reached near confluence by incubation with $0.05 \%$ trypsin-ethylenediamine tetraacetic acid. The cells were pelleted by centrifugation and resuspended in DMEM. Cells $\left(5 \times 10^{7}\right.$ per site $)$ were implanted subcutaneously into the right axilla of each mouse.

\section{Method of administration and dosing}

Fifty mice were randomly allocated to a control, phosphatebuffered saline, ND, ND-DOX, or free doxorubicin group ( $\mathrm{n}=10$ in each group). Animals in group 1 served as controls. The animals in group 2 received intraperitoneal injections of phosphate-buffered saline (1.0 mL, pH 7.4). Animals in group 3 received injections of NDs $(0.67 \mathrm{~mL}, 2.1 \mathrm{mg} / \mathrm{mL}$, suspended in phosphate-buffered saline to achieve the final concentration). Animals in group 4 received injections of doxorubicin ( $100 \mu \mathrm{g}$ per $20 \mathrm{~g}$ body weight). Animals in group 5 received injections of ND-DOX $(1.43 \mathrm{mg} \mathrm{ND}+$ doxorubicin $100 \mu \mathrm{g}$ per $20 \mathrm{~g}$ body weight). All injections were administered 24 hours following tumor implantation, and continued once per week until the animal was euthanized. Body weight was recorded for every animal before an injection. No infection, impaired mobility, or markedly reduced food intake was observed. All NDs or ND-DOX used were sonicated for 30 minutes to ensure thorough dispersion before use.

\section{Histopathological examination ex vivo}

The animals were observed after inoculation until they were euthanized. Two animals were randomly selected from each group for harvesting of tumor, liver, kidney, and spleen tissue. The harvested tissues were stored in $10 \%$ formaldehyde solution for pathological analysis. The tissues were dehydrated, processed routinely into paraffin, and embedded individually in paraffin blocks, and sectioned into $4 \mu \mathrm{m}$ thickness. The sections from each tissue sample were then stained with hematoxylin and eosin (H\&E) and analyzed by a pathologist using fluorescence microscopy.

\section{Results and discussion}

Effect of $\mathrm{pH}$ on doxorubicin release and adsorption

Characteristic surface groups on NDs include carboxyl, hydroxyl, and keto groups as well as anhydrides and lactones. ${ }^{31}$ NDs $(140 \mathrm{~nm})$ were prepared as described previously ${ }^{28,32}$ to form carboxylated NDs. Functional tight binding-based density simulations indicate that the interparticle interactions in NDs depend on the charges on the surface facets, leading to preferentially ordered self-assembled ND agglutinates. ${ }^{33,34}$ For the functionalized NDs used in drug binding, it is the ionizable groups on the ND surface rather than intrinsic electrostatic forces that dominate the interaction between NDs and charged molecules like doxorubicin hydrochloride. ${ }^{35}$ With the NDs being negatively charged $\left(\mathrm{ND}-\mathrm{COO}^{-}\right)$and the doxorubicin ions being positively charged $\left(\mathrm{NH}_{3}^{+}\right)$, one needs to consider variations in the protonation equilibrium on the surface sites of the NDs and the amine group on doxorubicin as determined by solvent cations or anions.

Adsorption of doxorubicin onto NDs was investigated at $\mathrm{pH}$ values between 3 and 10 (Figure 1A). The adsorption capacity gradually increased with increasing $\mathrm{pH}$. The NDs showed optimal adsorption capacity for doxorubicin in sodium hydroxide solution with a $\mathrm{pH}$ of 10 (final solution, $\mathrm{pH}$ 7.56). At a $\mathrm{pH} \geq 11$, the structure of doxorubicin changed (Figure S1), with a wavelength shift from $480 \mathrm{~nm}$ to $550 \mathrm{~nm}$. Therefore, ND-DOX was prepared in a sodium hydroxide solution at $\mathrm{pH} 10$ (final solution, $\mathrm{pH}$ 7.56). This would result in ionized $\mathrm{NH}_{3}^{+}$because doxorubicin is a weak base with a $\mathrm{pK}_{\mathrm{b}}$ of 8.3 and would associate with $\mathrm{COO}^{-}$on the NDs via electrostatic interaction. Therefore, formation of the ND-DOX complexes may be the result of electrostatic forces, hydrogen bonding, and Van der Waals forces between protonated amines on the doxorubicin molecules and the deprotonated carboxylic acid groups on ND.

It is well known that efficient drug release from a carrier system is a prerequisite for therapeutic activity of most macromolecular anticancer conjugates. Incorporation of acid sensitivity between the drug and carrier enables the release of an active drug from the carrier into tumor tissue, either in slightly acidic extracellular fluids (tumor environment, $\mathrm{pH} 6.5$ ) or after endocytosis in the endosomes (pH 5-6) or lysosomes (pH 4-5) of cancer cells. ${ }^{36}$ For these reasons, the drug release behavior of ND-DOX was investigated under simulated physiological conditions (phosphate-buffered saline, $\mathrm{pH}$ 7.4) and an acidic environment (phosphate-buffered saline, $\mathrm{pH} 5.5$ [an acidic endosome environment] and $\mathrm{pH} 6.5$ [a simulated tumor environment]) at $37^{\circ} \mathrm{C}$ to assess the feasibility of using ND-DOX as an anticancer drug delivery system. As shown in Figure 1B, the rate and amount of doxorubicin released from ND-DOX were dependent on the $\mathrm{pH}$ of the medium. ND-DOX showed a slightly more rapid release of doxorubicin 
A
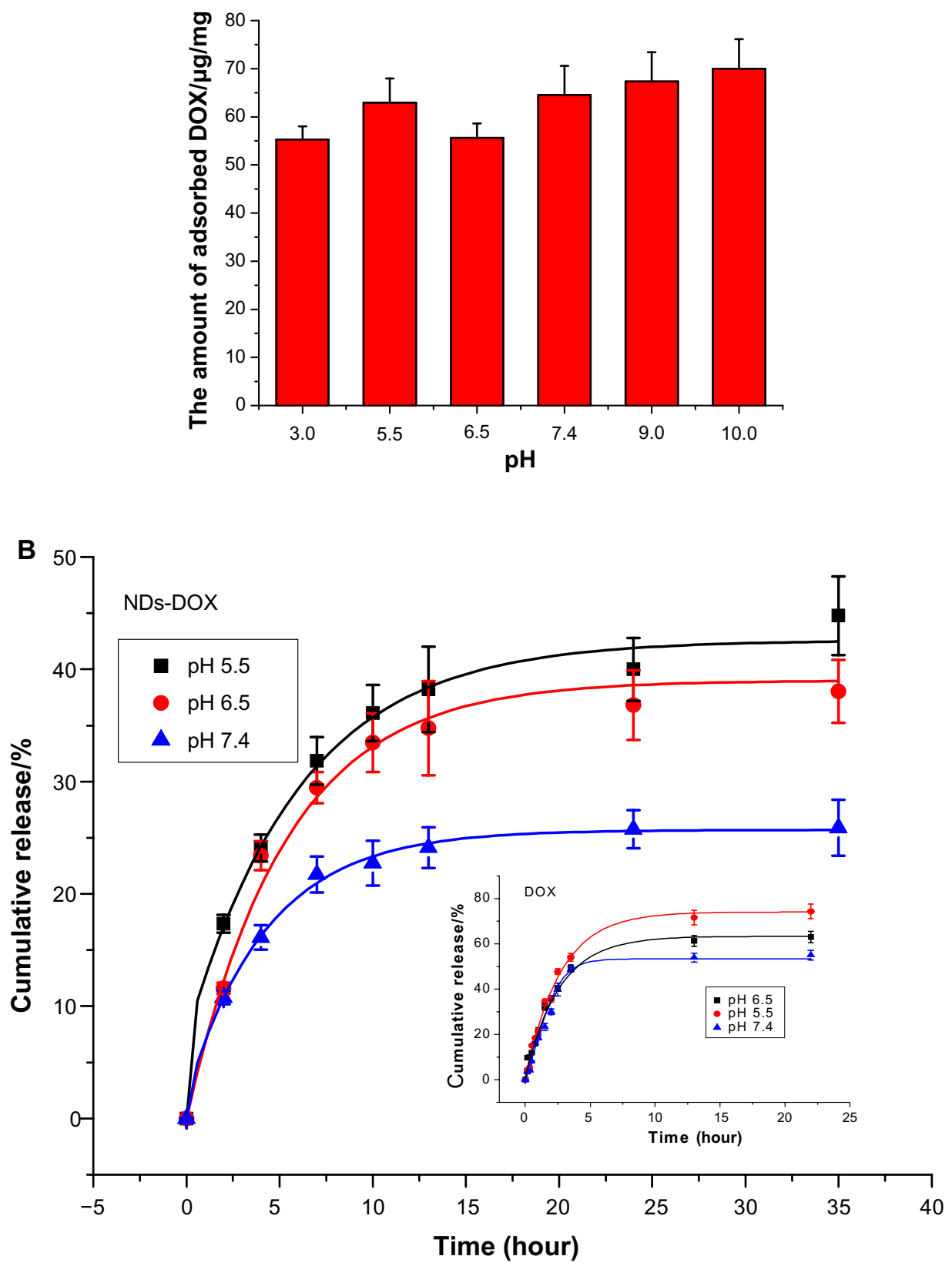

Figure I Effect of $\mathrm{pH}$ on adsorption and release of doxorubicin by NDs. (A) Variation of doxorubicin adsorbed onto NDs with pH. (B) Doxorubicin release profile for ND-DOX at $37^{\circ} \mathrm{C}$ (inset shows release profile for free doxorubicin).

Abbreviations: NDs, nanodiamond particles; ND-DOX, nanodiamond-doxorubicin complex; DOX, free doxorubicin.

at $\mathrm{pH} 5.5$ and $\mathrm{pH} 6.5$ than at $\mathrm{pH} 7.4$. At $\mathrm{pH} 5.5$ and $\mathrm{pH} 6.5$, ND-DOX released about $24 \%$ and $23 \%$, respectively, of the conjugated doxorubicin during the first 4 hours, and about $45 \%$ and $38 \%$, respectively, by 35 hours; however, at $\mathrm{pH} 7.4$, ND-DOX released only $16 \%$ of doxorubicin in the first 4 hours and less than $26 \%$ by 35 hours. These results indicate that the amount of doxorubicin released from ND-DOX was governed by an acidic environment. The slow doxorubicin release from ND-DOX observed at $\mathrm{pH} 7.4$, which mimics the physi- ological environment of the bloodstream, ensures that minimal doxorubicin is released from ND-DOX in the circulation. It is expected that ND-DOX would accumulate preferentially in tumor tissue via the enhanced permeability and retention effect. Once in the tumor tissue, these ND-DOX will be internalized by tumor cells, largely via clathrin-dependent endocytosis (Figure S2A), and will be located in the cytoplasm, where doxorubicin detached from FND-DOX could migrate and enter the nucleus to interact with DNA. ${ }^{30}$ As seen in the 
inset of Figure 1B, release of unmodified doxorubicin plateaus by approximately 4 hours at $\mathrm{pH} 7.4$ and by 6 hours at $\mathrm{pH} 6.5$ and 5.5, with a maximum release of $54 \%$ at $\mathrm{pH} 7.4,61 \%$ at $\mathrm{pH} 6.5$, and $72 \%$ at $\mathrm{pH}$ 5.5. In comparison with ND-DOX, free doxorubicin can damage cells in a normal physiological environment, causing serious side effects.

\section{Characteristics of ND-DOX}

Based on the optimized conditions described above, adsorption of doxorubicin onto NDs was maximal at $\mathrm{pH}$ 10.0, so we prepared ND-DOX in sodium hydroxide with a $\mathrm{pH}$ of 10.0. The amount of doxorubicin adsorbed onto ND was approximately $70.0 \pm 2.3 \mu \mathrm{g} / \mathrm{mg}\left(7.29 \times 10^{16}\right.$ molecules per $\mathrm{mg}$ ), which is about twice that of the adsorption rate in distilled water. ${ }^{28}$ Dynamic light scattering was used to determine the average size and zeta potential of the NDs and ND-DOX. Characterization of carboxylated ND revealed that the NDs form average cluster sizes of nearly $141 \pm 1.3 \mathrm{~nm}$. ND-DOX had an average size of about $165 \pm 1.7 \mathrm{~nm}$ at dilute concentrations and was dispersible in deionized water. The dynamic light scattering data also showed that ND-DOX had a higher zeta potential $(-21.8 \pm 1.9 \mathrm{mV})$ than carboxylated ND, which was indicative of surface binding. Further, the ND and NDDOX particles showed a narrow size distribution, with polydispersity indices ranging from 0.078 to 0.103 , calculated at $25^{\circ} \mathrm{C}$ with a $90^{\circ}$ scattering angle by cumulative analysis using the Zetasizer Nano-ZS90 software (Malvern Instruments Ltd). Compared with pristine NDs, the polydispersity index of ND-DOX was slightly increased, implying that aggregation of ND-DOX was enhanced. However, such a low polydispersity index range indicates that ND-DOX undergoes only minor aggregation and should not be prone to precipitation, which is the advantage of ND-DOX delivery.

Transmission electron microscopy was used to further verify formation of the ND-DOX complex. Figure 2A shows typical images before and after coating with doxorubicin. Clear structures were visible in samples of ND alone, and the structures were markedly less visible after formation of NDDOX (Figure 2A). It can be seen that ND-DOX was different from pristine NDs. The thickness of ND-DOX was increased, and the degree of aggregation and particle size became slightly larger than for ND alone. These findings are consistent with those from dynamic light scattering. Formation of ND-DOX was confirmed further by Fourier transform infrared (FTIR) analysis. The FTIR peaks, indicated with the arrows in Figure 2B(3), can be clearly assigned to the drug. As a comparison, the FTIR spectrum for pure doxorubicin is shown in Figure $2 \mathrm{~B}(2)$. In addition to the readily formed
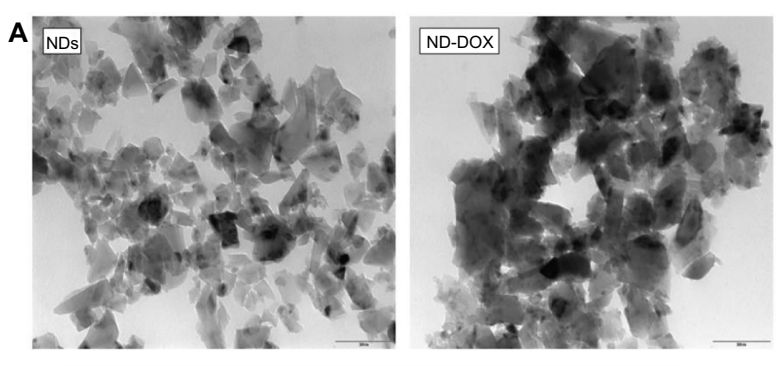

B

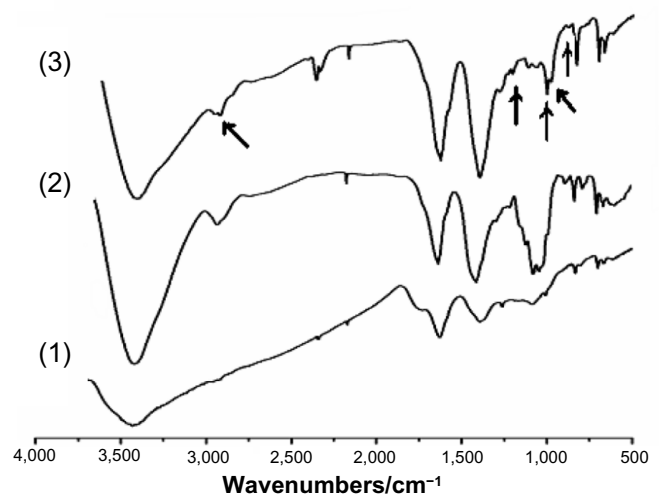

Figure 2 Characteristics of ND-DOX. (A) Typical transmission electron microscopic images before and after coating with doxorubicin, respectively. The scale bars represent $200 \mathrm{~nm}$. (B) Fourier transform infrared spectra confirming NDs adsorbed with doxorubicin. NDs, doxorubicin, and ND-DOX are shown in Figure $B(I-3)$, respectively. The arrows in Figure $B(3)$ indicate the presence of doxorubicin on NDs. Abbreviations: NDs, nanodiamond particles; ND-DOX, nanodiamond-doxorubicin complex.

aqueous ND suspensions, the nature of the NDs can be seen in the rich presence of functional $-\mathrm{OH}$ and $-\mathrm{COOH}$ groups in the FTIR spectrum, as shown in Figure 2B(1).

\section{Sustained in vitro drug release from ND-DOX into HepG2 cells}

In order to determine the characteristics of controlled drug release from ND-DOX in vitro, HepG2 cells were treated with FND-DOX or doxorubicin alone in DMEM with $10 \%$ fetal bovine serum and incubated over defined periods of time. As early as one hour after treatment, doxorubicin had entered the cell nucleus (Figure 3B). However, little FND-DOX reached the treated cells for this time. Three hours after treatment, FND-DOX had entered the cells and could be visualized in the cytoplasm, where orange-yellow fluorescence was visible due to merging of the red fluorescence from FND and the green fluorescence from doxorubicin (Figure 3C). More FNDDOX was seen with the passage of time, and this is confirmed quantitatively in Figure S4. Bright green fluorescence was seen to appear, indicating the doxorubicin molecules released from FND-DOX at 5 hours (Figure 3C). At 16 hours, separate green and red fluorescence signals from doxorubicin and the FND particles, respectively, were seen (Figure 3C), implying 

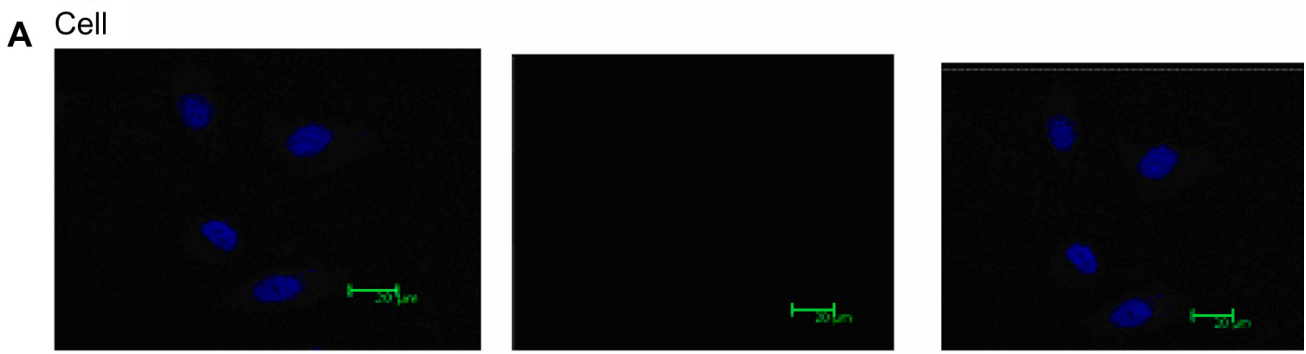

DOX $1 \mathrm{~h}$

B
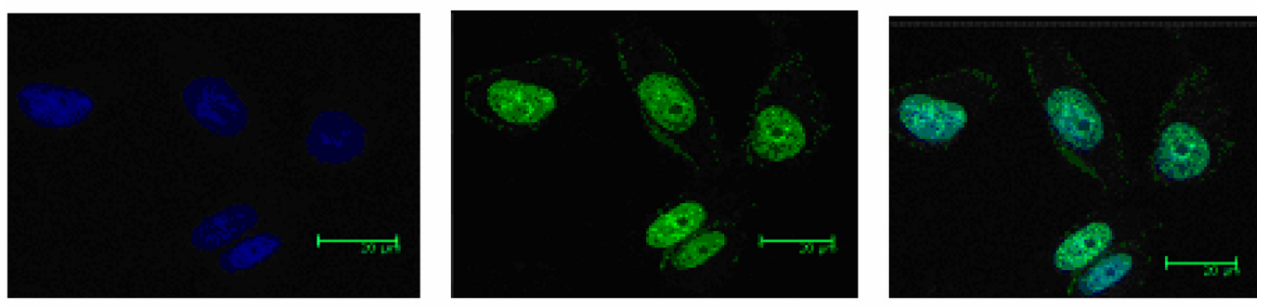

FND-DOX
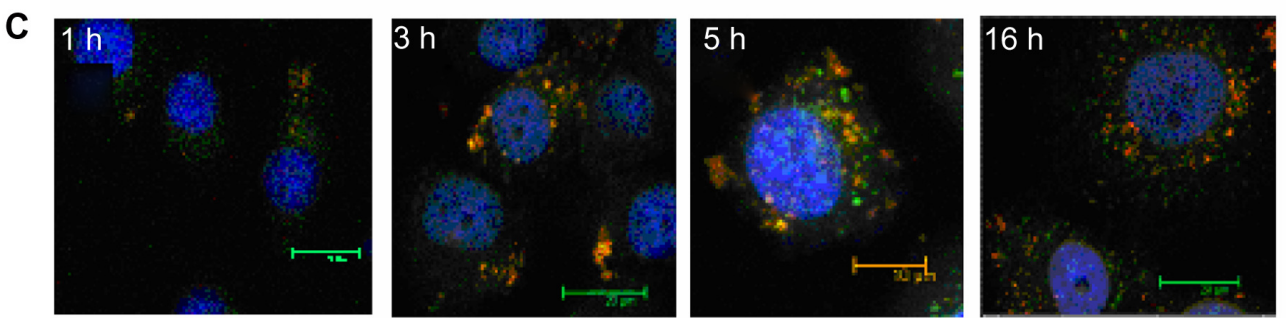

Figure 3 Characteristics of controlled drug release from FND-DOX in HepG2 cells in vitro. The cell nuclei are stained with $\mathrm{H} 33258$ (blue), doxorubicin is green (excitation wavelength $488 \mathrm{~nm}$ and emission wavelength $560-590 \mathrm{~nm}$ ) and FND is red (excitation wavelength $543 \mathrm{~nm}$ and emission wavelength 630-700 nm). Bright orange represents colocalization of doxorubicin (green) and FND (red). (A and B) Images in the left column show the cell nucleus dyed with H33258; the middle column shows cell autofluorescence and doxorubicin fluorescence; and the right column shows the merge of the two previous images. (A) Images for the control group, (B) images for cells treated with doxorubicin, and $(\mathbf{C})$ merged images for cells treated with FND-DOX over time.

Abbreviations: FND-DOX, fluorescence nanodiamond-doxorubicin complex; FND, red fluorescence nanodiamond; h, hour; DOX, free doxorubicin.

that most of the doxorubicin was released from FND-DOX around the nucleus. These results demonstrate that ND-DOX is capable of mediating controlled drug release and has a slower and more sustained drug release profile.

The MTT (3-[4,5-dimethylthiazol-2-yl]-2,5 diphenyl tetrazolium bromide) test was used to determine whether or not ND-DOX is effective in causing cell damage. The results show that cell viability was significantly decreased in the presence of ND-DOX and in a concentration-dependent manner (Figure S2). Hence, we hypothesized that FND-DOX was transported into an acidic endosomal environment via endocytosis. The low $\mathrm{pH}$ induced release of doxorubicin, which entered the nucleus and became intercalated into DNA to inhibit DNA replication and cell growth.

ND-DOX can efficiently deliver doxorubicin into HeLa cells via a clathrin-dependent endocytosis pathway. ${ }^{30} \mathrm{In}$ contrast, the uptake of doxorubicin occurs through an energyindependent passive diffusion mechanism. ${ }^{30} \mathrm{~A}$ similar result was shown for FND-DOX in HepG2 cells (Figure S3).
Time-dependent measurements revealed that the fluorescence intensity reached a plateau at about 10 hours for FND-DOX and at 4 hours for free doxorubicin (Figure S4). Curve fitting by a single exponential function showed that the rate constants for internalization of doxorubicin were $0.33 \pm 0.059$ per hour for FND-DOX and 1.21 \pm 0.066 per hour for free doxorubicin. The internalization rate for FND-DOX was about 3.7 times longer than for doxorubicin alone. This may be the result of the different uptake pathways utilized by FND-DOX and free doxorubicin.

\section{In vivo inhibition of tumor growth and improved survival using ND and ND-DOX}

To determine whether ND-DOX is an appropriate drug delivery vehicle in vivo, tumors were first allowed to inoculate with H22 liver cancer cells to form tumors in mice. The tumor-bearing mice were treated weekly with ND-DOX, doxorubicin, ND, or phosphate-buffered saline 24 hours post 
tumor inoculation. We analyzed long-term tumor burden until the mice were euthanized. During the observation period, we found that treatment with bare NDs significantly improved the life span of the mice compared with doxorubicin alone. Our results show that doxorubicin, ND, and ND-DOX effectively inhibited tumor growth compared with control and phosphatebuffered saline (Figure 4A and B). Additionally, doxorubicin resulted in survival curves that were similar to those for mice treated with NDs, and the survival rate improved significantly in mice treated with ND-DOX in comparison with mice treated with free doxorubicin (Figure 4C and D). The median survival time is shown in Table 1 . The median survival time means that the survival rate was $50 \%$ corresponding to the survival time. Table 1 shows that the median survival time in mice treated with ND-DOX was 28.2 days versus 20.5 or 20 days for mice treated with doxorubicin or NDs, respectively, and survival time in the group treated with phosphate-buffered saline was similar to that of the control group. The life span (\%) for the control groups was defined as follows:

$$
\text { Life span }(\%)=\left(\frac{\mathrm{Md}_{\text {sample }}}{M d_{\text {control }}}-1\right) \times 100 \%
$$

where $\mathrm{Md}_{\text {sample }}$ is the median survival time for mice treated with phosphate-buffered saline, ND, doxorubicin, and NDDOX, and $\mathrm{Md}_{\text {control }}$ is the median survival time for tumorbearing control mice. The data in Figure 4D show that the life span was $57.5 \%$ for the ND-DOX group, $11.73 \%$ for the ND group, $14.5 \%$ for the doxorubicin group, and $1.12 \%$ for the phosphate-buffered saline group. Therefore, treatment with NDs and ND-DOX significantly improved the survival rate when compared with the phosphate-buffered saline-treated and control tumor-bearing mice (Figure 4C and D). The survival rate of mice treated with ND-DOX was four
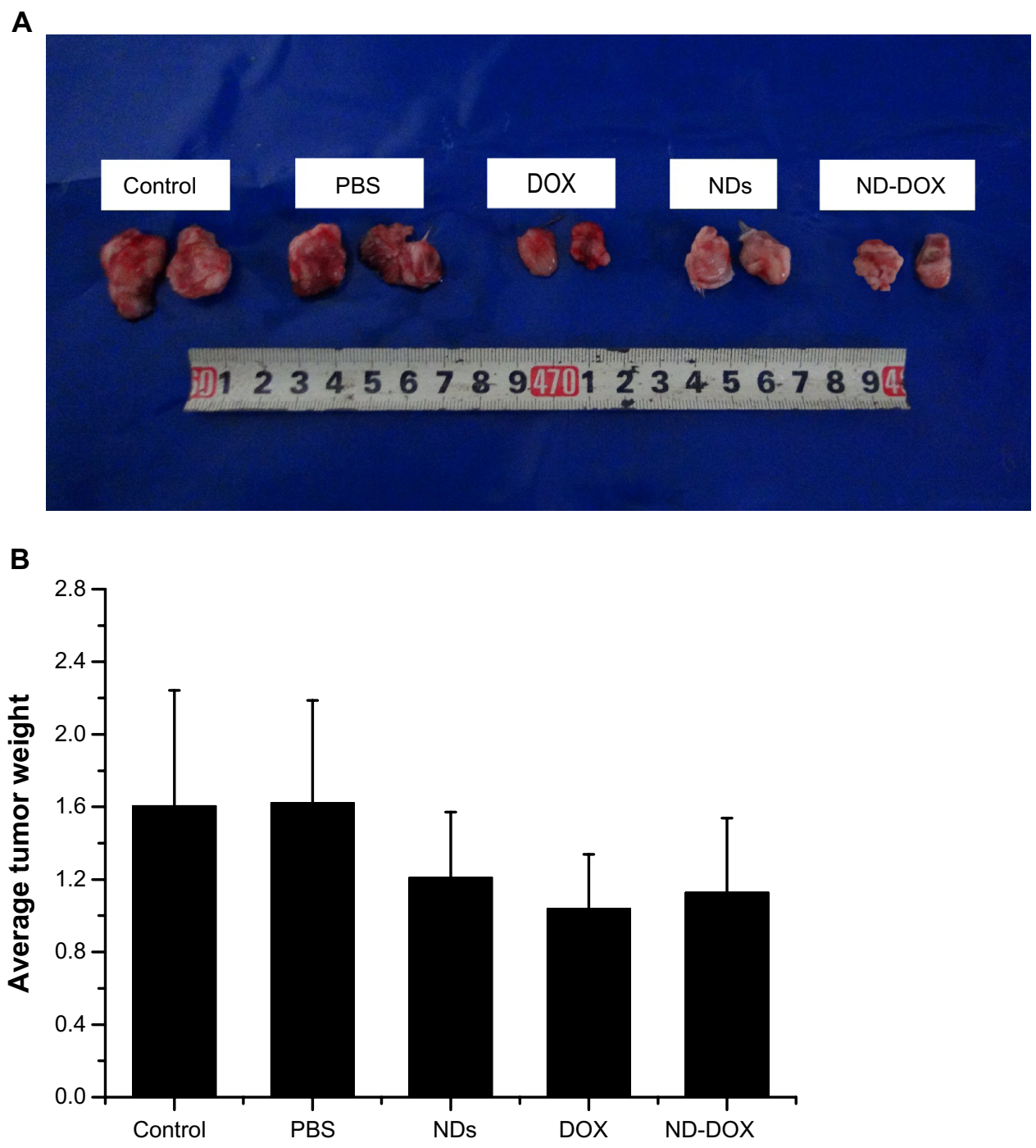

Figure 4 (Continued) 


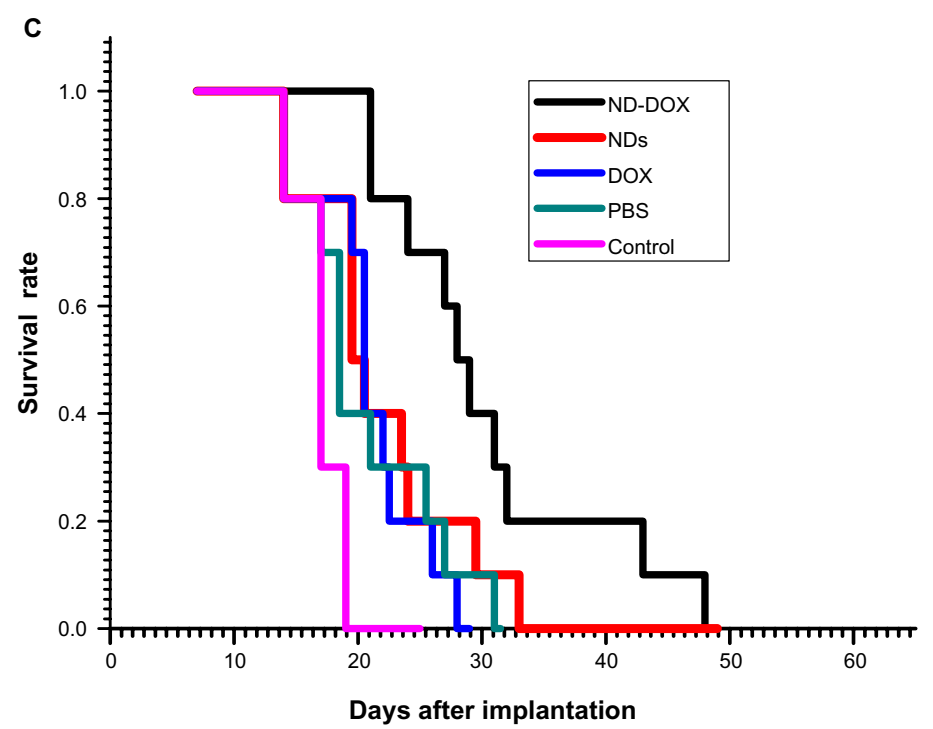

D
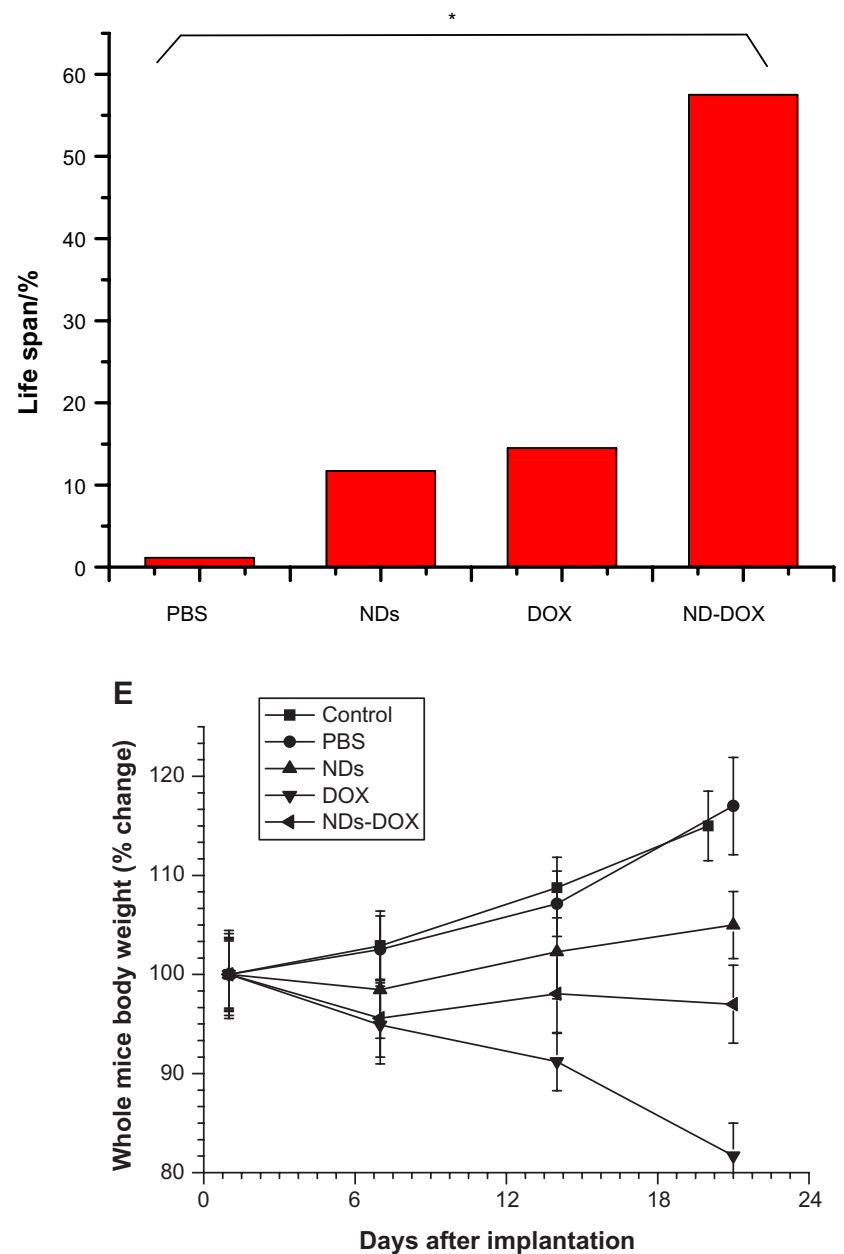

Figure 4 Inhibition of tumor growth and improved survival probability using NDs and ND-DOX compared with doxorubicin in vivo. (A) Representative images of tumors from treated mice. (B) Average tumor weight analysis post mortem for mice treated with phosphate-buffered saline $(n=9)$, doxorubicin $100 \mathrm{mg} / 20 \mathrm{~g}$ mouse body weight $(n=6)$, ND-DOX ( $100 \mathrm{mg}$ of doxorubicin equivalent per $20 \mathrm{~g}$ mouse body weight, $\mathrm{n}=6)$, or NDs ( $1.42 \mathrm{mg}$ per $20 \mathrm{~g}$ mouse body weight, $\mathrm{n}=8$ ) via intraperitoneal injection every 7 days. Control group $(n=10)$. (C) Kaplan-Meier survival plot for tumor-bearing mice treated with phosphate-buffered saline $(n=10)$, doxorubicin ( $100 \mathrm{mg} / 20 \mathrm{~g}$ mouse body weight, $\mathrm{n}=10$ ), ND-DOX ( $100 \mathrm{mg}$ of doxorubicin equivalent per $20 \mathrm{~g}$ mouse body weight, $\mathrm{n}=10$ ), or NDs ( $1.42 \mathrm{mg}$ per $20 \mathrm{~g}$ of mouse body weight, $\mathrm{n}=10$ ) via intraperitoneal injections every 7 days. (D) Plot of median (Md) survival in treated tumor-bearing mice compared with control group $(* P<0.05)$. (E) Average whole body weight analysis of tumorbearing mice treated with phosphate-buffered saline $(n=5)$, doxorubicin $100 \mathrm{mg} / 20 \mathrm{~g}$ mouse body weight $(n=5)$, ND-DOX ( $100 \mathrm{mg}$ of doxorubicin equivalent per $20 \mathrm{~g}$ mouse body weight, $n=5)$, or NDs ( $1.42 \mathrm{mg}$ per $20 \mathrm{~g}$ of mouse body weight $(n=5)$ via intraperitoneal injection every 7 days. Data are shown as the mean \pm standard deviation. Abbreviations: DOX, free doxorubicin; NDs, nanodiamond particles; ND-DOX, nanodiamond-doxorubicin complex; PBS, phosphate-buffered saline. 
Table I Median survival time (Md) in tumor-bearing mice treated with phosphate-buffered saline, doxorubicin, ND-DOX, or NDs via intraperitoneal injection every 7 days

\begin{tabular}{llllll}
\hline Groups & Control & PBS & ND & DOX & ND-DOX \\
\hline Md/days & 17.9 & 18.1 & 20.0 & 20.5 & 28.2 \\
\hline
\end{tabular}

Note: The median survival time means that the survival rate was $50 \%$ corresponding to the survival time

Abbreviations: NDs, nanodiamond particles; PBS, phosphate-buffered saline; ND-DOX, nanodiamond-doxorubicin complex.

times that of mice treated with doxorubicin, and survival in the ND-treated group was close to that of the doxorubicintreated group. However, Chow et al reported that NDs with a size of $45.1 \mathrm{~nm}$ cannot significantly inhibit tumor growth alone, and the probability of survival after treatment with bare NDs has not been reported to date. ${ }^{26}$ This difference may be affected by the size of the ND used as well as use of different tumor models. This reflects the biological effects of nanoparticle size. Verma and Stellacci have reported, the cellular uptake of nanoparticles is related to nanoparticle size. ${ }^{37}$ For example, smaller gadolinium-containing nanoparticles $(400 \mathrm{~nm})$ have a stronger cell cycle-promoting effect than larger ones $(1,440 \mathrm{~nm}$ and $1,370 \mathrm{~nm}){ }^{22}$ The kinetics of uptake as well as the saturation concentration have been shown to vary between different-sized gold nanoparticles (eg, 14 nm,
$50 \mathrm{~nm}$, and $74 \mathrm{~nm}) .{ }^{38-40}$ Our results showing that ND-DOX effectively inhibits tumor growth and improves the probability of survival are consistent with the literature. ${ }^{26}$

Measurements of mouse body weight were recorded over time. Figure 4E shows a typical set of body weight data. It should be noted that there were no significant weight changes in the mice after treatment with ND or NDDOX compared with control mice and those treated with phosphate-buffered saline. However, the doxorubicin-treated groups showed weight loss with the passage of time. Multiple injections of the bare NDs did not have obvious toxicological effects, suggesting that NDs are biocompatible. Several biocompatibility studies of the particles in vitro with various cell lines have shown that ND is among the least toxic of all the carbon-based nanomaterials tested so far. ${ }^{41,42}$ The excellent biocompatibility of the carbon-based nanomaterial (ie, NDs) has been demonstrated recently, ${ }^{7,21,43,44}$ which makes it superior to other nanoparticles, such as nanosilica, which has very recently been shown to cause liver injury after continuous intraperitoneal injection ${ }^{44}$ in vivo. ND therefore serves as an effective drug carrier in vivo. Furthermore, the ND-DOX treatment decreased the toxicity of doxorubicin, as it avoids the weight loss that occurs with doxorubicin alone.

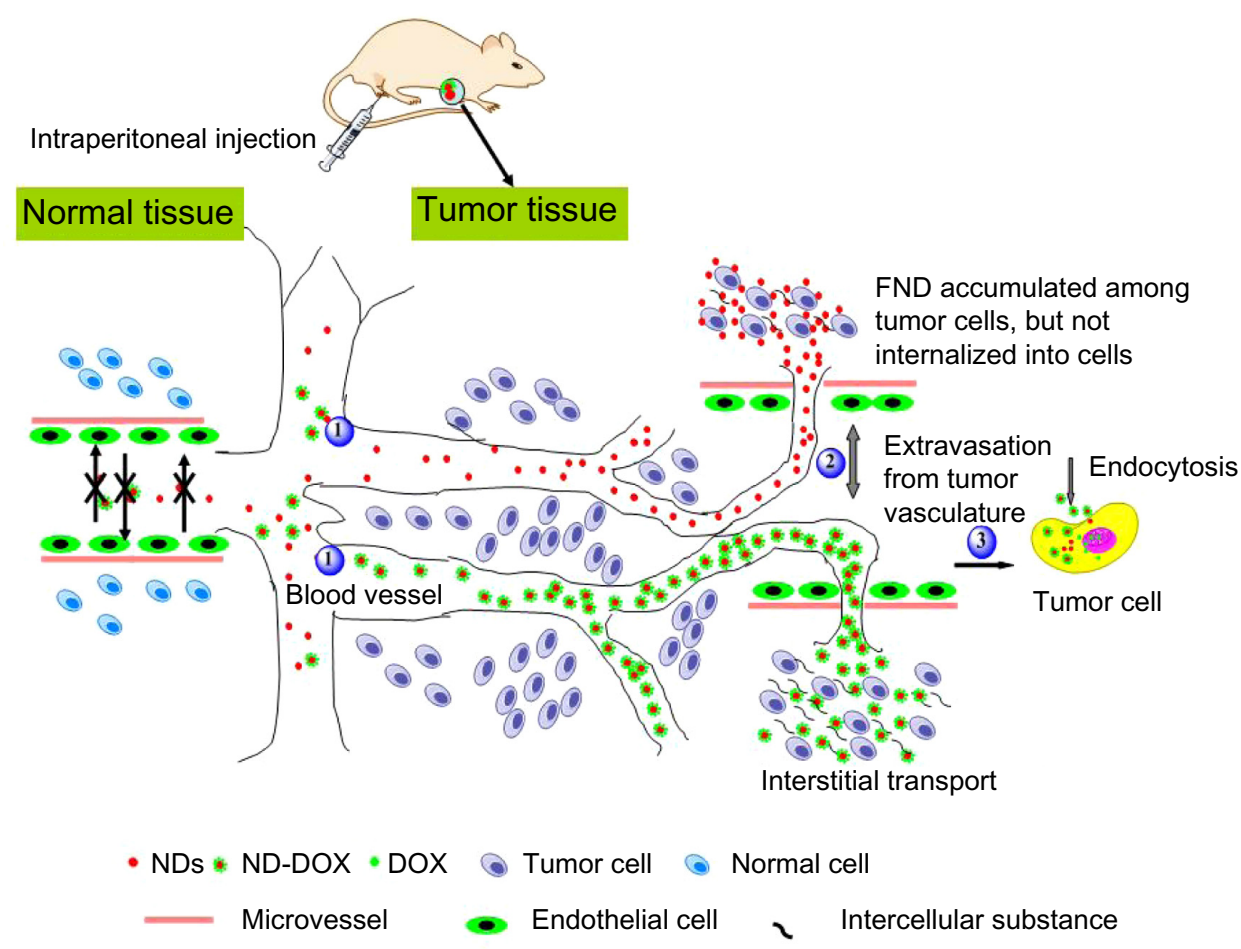

Figure 5 Transport of NDs and ND-DOX nanoparticles in normal and tumor tissue. 1) Transport in tumor vasculature. 2) Extravasation from tumor vasculature via enhanced permeability and retention effect. 3) Endocytosis of ND-DOX nanoparticles by cells, and then doxorubicin release from the ND-DOX complex, followed by entering the nucleus, intercalating into DNA to inhibit DNA replication and cell growth.

Abbreviations: DOX, free doxorubicin; NDs, nanodiamond particles; ND-DOX, nanodiamond-doxorubicin complex; FND, red fluorescence nanodiamond. 

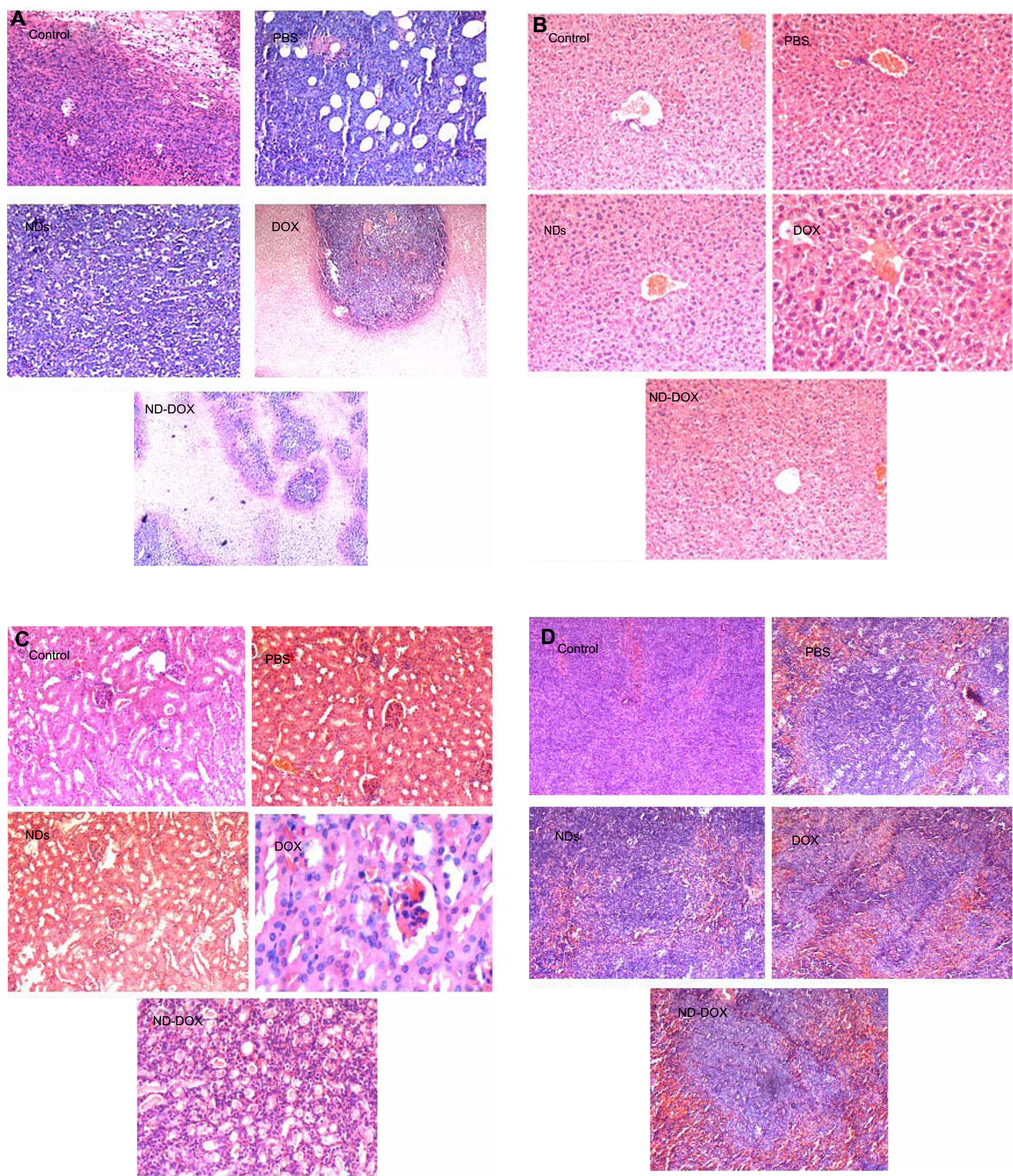

Figure 6 Representative mouse tissue sections stained with hematoxylin and eosin. Long-term toxicity was evaluated in mice injected with phosphate-buffered saline for 19 days, ND for 20 days, ND-DOX for 32 days, doxorubicin for 20 days, and a control group with tumor growth for 17 days. With the exception of the control group, the groups were injected intraperitoneally once a week. Animals in group 2 received injections of phosphate-buffered saline ( $1.0 \mathrm{~mL}$, $\mathrm{pH} 7.4$ ). Animals in group 3 received injections of $\mathrm{ND}(0.67 \mathrm{~mL}, 2.1 \mathrm{mg} / \mathrm{mL}$, suspended in phosphate-buffered saline to achieve the final concentration). Animals in group 4 received injections of doxorubicin (100 $\mu \mathrm{g} / 20 \mathrm{~g}$ body weight). Animals in group 5 received injections of ND-DOX ( $1.43 \mathrm{mg}$ ND/FND $100 \mu \mathrm{g}$ doxorubicin/20 g body weight). All injections were administered once, 24 hours after tumor inoculation. Thereafter, all intraperitoneal injections were done once per week until the specified time. Mouse body weight was monitored before each injection. (A) Tumor tissue, (B) liver tissue, (C) kidney tissue, and (D) spleen tissue.

Abbreviations: DOX, free doxorubicin; NDs, nanodiamond particles; ND-DOX, nanodiamond-doxorubicin complex; FND, red fluorescence nanodiamond; PBS, phosphatebuffered saline. 
These interesting results are thought to be related to the enhanced permeability and retention effect regarding macromolecules. ${ }^{45,46}$ The tumor microvasculature is discontinuous or "leaky", with gaps typically $100-400 \mathrm{~nm}$ in diameter, while the normal tissue microvascular endothelial cell wall connections are tight, with gaps generally being less than $10 \mathrm{~nm}$ in diameter. ${ }^{27}$ By exploiting this characteristic of tumor vasculature, ND and ND-DOX can preferentially target tumor sites.

There was abundant angiogenesis in tumor tissue, and ex vivo tumor angiogenesis imaging of representative tumor sections of mouse H22 liver cancer cells from the control, phosphate-buffered saline, ND, doxorubicin, and ND-DOX group are shown in Figure S5 (black arrows show the location of tumor angiogenesis). We believe that the nanoparticles tended to accumulate at the tumor site due to the enhanced permeability and retention effect, which is schematically illustrated in Figure 5. We propose that ND-DOX enters into the tumor site, and is taken up by cells where they release active drug, which enters the nucleus and intercalates into DNA, thereby inhibiting DNA replication and cell growth. Analogously, when NDs reach the tumor site, they enter into the tumor blood vessels, leading to decreased nutrient uptake by the tumor and subsequent tumor necrosis and slowed tumor growth (Figure 5). Hence, ND alone can also suppress tumor growth to increase the probability of survival in tumorbearing mice. In summary, ND-DOX prolongs the life span of tumor-bearing mice, targeting the tumor site while causing little harm to adjacent normal cells. Thus, the delivery of chemotherapeutics by NDs appears to improve tumor targeting in vivo and can serve as an effective drug delivery option to overcome limitations of free doxorubicin. It is important to understand that NDs and ND-DOX are indeed targeted to the tumor site once injected into animals, so further detailed studies are planned.

\section{Histological analysis}

We evaluated the effects of ND, ND-DOX, and free doxorubicin on tumor, kidney, liver, and spleen tissues from treated mice by histological analysis (Figure 6). All tested mice, including the controls, showed no tumor formation in the liver, spleen, or kidney. No apparent histopathological abnormalities or lesions related to the treatments were observed in the spleen for any of the mice. No obvious histological lesion was observed in the major organs, such as the liver or kidney, of mice treated with ND or ND-DOX. Histological analysis revealed that the liver was almost normal in mice treated with ND or ND-DOX. In the control group, inflammation was not obvious, and a portion of the liver cells showed mild hydropic degeneration. In the phosphate-buffered saline group, the central venous region showed congestion, with minimal inflammatory cell infiltration. In the doxorubicin-treated group, the lobular architecture of the liver was normal and showed the nucleus of the hepatocytes was enlarged. Furthermore, a small number of liver cells were necrotic. The kidney was minimally affected by NDs and ND-DOX, with the kidney cortex exhibiting scattered small focal areas of vascular dilatation and congestion. In kidneys from mice treated with doxorubicin, tubular cells showed turbidity and swelling, and the glomerulus was atrophied. In addition, both the cortical vasculature and the glomerular capillaries were congested. Hence, neither ND nor ND-DOX induced any significant changes in major organs such as the kidney, liver, and spleen, while serious injury to the kidney and liver was seen in mice treated with free doxorubicin (Figure 6). Extensive areas of necrosis and infiltration of inflammation cells at the tumor site caused by doxorubicin alone were observed 20 days posttreatment. ND and ND-DOX also induced tumor necrosis by 20 and 52 days post-treatment, respectively. There was tumor infiltration into the adipose tissue in control mice and mice treated with phosphate-buffered saline 19 days post treatment. Hence, doxorubicin alone had serious side effects, and mice left untreated developed cancer metastasis.

\section{Conclusion}

Our experiments demonstrate that, when compared with free doxorubicin, ND-DOX has a sustained release and passive targeting effect, and can effectively destroy tumor tissue without affecting the liver, kidney, or spleen. In addition, ND-DOX markedly improved the life expectancy of tumor-bearing mice, which was four times that of mice treated with free doxorubicin. Hence, ND-DOX may not only have reduced toxicity, but can also effectively inhibit tumor growth. In the course of this study, we found an interesting phenomenon, ie, NDs alone could also suppress tumor growth, achieving a survival rate close to that in mice treated with free doxorubicin. To the best of our knowledge, such an interesting result has not been reported. This promising foundation for continued development and potential clinical application of NDs warrants further research. Future investigation of targeted chemotherapeutics using drug-loaded NDs will have far reaching significance.

\section{Acknowledgments}

The authors are grateful to Professor HC Chang, Institute of Atomic and Molecular Sciences, Academia Sinica, Taiwan, for supply of the FND used in this study. This research 
is supported by the National Natural Science Foundation of China (21071091), Shanxi Science and Technology Development Program (20130313021-1), the Shanxi Provincial Natural Science Foundation (2009011012-3), and the Shanxi Scholarship Council of China (201011). The authors thank Gang Su, Wei Bai, and Lianshneg Ren for their assistance with this work.

\section{Disclosure}

The authors report no conflicts of interest in this work.

\section{References}

1. Nakamura E, Isobe H. Functionalized fullerenes in water. Acc Chem Res. 2003;36:807-815.

2. Lin Y, Taylor S, Li H, et al. Advances toward bioapplications of carbon nanotubes. J Mater Chem. 2004;14:527-541.

3. Bianco A, Kostarelos K, Partidos CD, Prato M. Biomedical applications of functionalised carbon nanotubes. Chem Commun. 2005;5:571-577.

4. Chen X, Tam UC, Czlapinski JL, et al. Interfacing carbon nanotubes with living cells. J Am Chem Soc. 2006;128:6292-6293.

5. Manna SK, Sarkar S, Barr J, et al. Single-walled carbon nanotube induces oxidative stress and activates nuclear transcription factor-KB in human keratinocytes. Nano Lett. 2005;5:1676-1684.

6. Lacerda L, Bianco A, Prato M, Kostarelos K. Carbon nanotubes as nanomedicines: from toxicology to pharmacology. Adv Drug Deliv Rev. 2006;58:1460-1470.

7. Schrand AM, Huang HJ, Carlson CJ, Schlager J, Osawa ES, Hussain ML. Are diamond nanoparticles cytotoxic? J Phys Chem B. 2007;111:2-7.

8. Yu SJ, Kang MW, Chang HC, Chen KM, Yu YC. Bright fluorescent nanodiamonds: no photobleaching and low cytotoxicity. $J$ Am Chem Soc. 2005;127:17604-17605.

9. Wang DX, Tong YL, Li YQ, Tian ZM, Cao RX, Yang BS. PEGylated nanodiamond for chemotherapeutic drug delivery. Diam Relat Mater. 2013;36:26-34.

10. Schrand AM, Dai L, Schlager JJ, Hussain SM, Osawa E. Differential biocompatibility of carbon nanotubes and nanodiamonds. Diam Relat Mater. 2007;16:2118-2123.

11. Zhang XY, Hu WB, Li J, Tao L, Wei Y. A comparative study of cellular uptake and cytotoxicity of multi-walled carbon nanotubes, graphene oxide, and nanodiamond. Toxicol Res. 2012;1:62-68.

12. Puzyr AP, Baron AV, Purtov KV, et al. Nanodiamonds with novel properties: a biological study. Diam Relat Mater. 2007;16:2124-2128.

13. Yuan Y, Chen YW, Liu JH, Wang H, Liu Y. Biodistribution and fate of nanodiamonds in vivo. Diam Relat Mater. 2009;18:95-100.

14. Chang HC. In: Ho D, editor. Nanodiamonds. New York, NY, USA: Springer; 2010.

15. Fu CC, Lee HY, Chen K, et al. Characterization and application of single fluorescent nanodiamonds as cellular biomarkers. Proc Natl Acad Sci USA. 2007;104:727-732.

16. Zhang BL, Li YQ, Fang CY, et al. Receptor-mediated cellular uptake of folate-conjugated fluorescent nanodiamonds: a combined ensemble and single-particle study. Small. 2009;5:2716-2721.

17. Li YQ, Zhou XP. Transferrin-coupled fluorescence nanodiamonds as targeting intracellular transporters: an investigation of the uptake mechanism. Diam Relat Mater. 2010;1:1163-1167.

18. Fu YJ, An N, Zheng SH, Liang AH, Li YQ. BmK CT-conjugated fluorescence nanodiamond as potential glioma-targeted imaging and drug. Diam Relat Mater. 2012;21:73-76.

19. Mohan N, Chen CS, Hsieh HH, Wu YC, Chang HC. In vivo imaging and toxicity assessments of fluorescent nanodiamonds in Caenorhabditis elegans. Nano Lett. 2010;10:3692-3699.
20. Rojas S, Gispert JD, Martin R, et al. Biodistribution of aminofunctionalized diamond nanoparticles. In vivo studies based on ${ }^{18} \mathrm{~F}$ radionuclide emission. ACS Nano. 2011;5:5552-5559.

21. Vaijayanthimala V, Cheng PY, Yeh SH, et al. The long-term stability and biocompatibility of fluorescent nanodiamond as an in vivo contrast agent. Biomaterials. 2012;33:7794-7802.

22. Service RF. Nanotechnology takes aim at cancer. Science. 2005;310: 1132-1134.

23. Peer D, Karp JM, Hong S, Farokhzad OC, Margalit R, Langer R. Nanocarriers as an emerging platform for cancer therapy. Nat Nanotechnol. 2007;2:751-760.

24. Sanhai WR, Sakamoto JH, Canady R, Ferrari M. Seven challenges for nanomedicine. Nat Nanotechnol. 2008;3:242-244.

25. Huang H, Pierstorff E, Osawa E, Ho D. Active nanodiamond hydrogels for chemotherapeutic delivery. Nano Lett. 2007;7:3305-3314.

26. Chow EK, Zhang XQ, Chen M, et al. Nanodiamond therapeutic delivery agents mediate enhanced chemoresistant tumor treatment. Sci Transl Med. 2011;3:73ra21.

27. Khaled G. Enhanced permeability and retention of macromolecular drugs in solid tumors: a royal gate for targeted anticancer nanomedicines. J Drug Target. 2007;15:457-464.

28. Li JX, Liu JC, Wang K, Yang XG. Gadolinium-containing bioparticles as an active entity to promote cell cycle progression in mouse embryo fibroblast NIH3T3 cells. J Biol Inorg Chem. 2010;15:547-557.

29. Matsumura Y, Maeda H. A new concept for macromolecular therapeutics in cancer chemotherapy: mechanism of tumoritropic accumulation of proteins and the antitumor agent Smancs1. Cancer Res. 1986;46: $6387-6392$.

30. Li YQ, Zhou XP, Wang DX, Yang BS, Yang P. Nanodiamond mediated delivery of chemotherapeutic drugs. J Mater Chem. 2011;21: 16406-16412.

31. Muggia FM. Doxorubicin-polymer conjugates: further demonstration of the concept of enhanced permeability and retention. Clin Cancer Res. 1999;5:7-8.

32. VonMaltzahn G, Park JH, Lin KY, et al. Nanoparticles that communicate in vivo to amplify tumour targeting. Nat Mater. 2011;10:545-552.

33. Wang J, Tian S, Petros RA, Napier ME, DeSimone JM. The complex role of multivalency in nanoparticles targeting the transferrin receptor for cancer therapies. J Am Chem Soc. 2010;132:11306-11313.

34. Lu J, Liong M, Li ZX, Zink JI, Tamanoi F. Biocompatibility, biodistribution, and drug-delivery efficiency of mesoporous silica nanoparticles for cancer therapy in animals. Small. 2010;6:1794-1805.

35. Rejmanova P, Kopecek J, Pohl J, Baudys M, Kostka V. Polymers containing enzymatically degradable bonds 8 . Degradation of oligopeptide sequences in N-(2-hydroxypropyl)methacrylamide copolymers by bovine spleen cathepsin B. Makromol Chem. 1983;184: 2009-2020.

36. Krueger A. New carbon materials: biological applications of functionalized nanodiamond materials. Chem Eur J. 2008;14:1382-1390.

37. Verma A, Stellacci F. Effect of surface properties on nanoparticle-cell interactions. Small. 2010;6:12-21.

38. Chithrani BD, Chan WCW. Elucidating the mechanism of cellar uptake and removal of protein-coated gold nanoparticles of different sizes and shapes. Nano Lett. 2007;7:1542-1550.

39. Chithrani BD, Ghazani AA, Chan WCW. Determining the size and shape dependence of gold nanoparticle uptake into mammalian cells. Nano Lett. 2006;6:662-668.

40. Schrand AM, Johnson J, Dai L, Hussain SM, Schlager JJ, Zhu L. Cytotoxicity and genotoxicity of carbon nanomaterials. In: Webster TJ, editor. Safety of Nanoparticles, Nanostructure Science and Technology. Morwell, Australia: Springer; 2009.

41. Xing Y, Xiong W, Zhu L, Osawa E, Hussin S, Dai L. DNA damage in embryonic stem cells caused by nanodiamonds. ACS Nano. 2011;5: 2376-2384.

42. Vaijayanthimala V, Tzeng YK, Chang HC, Li CL. The biocompatibility of fluorescent nanodiamonds and their mechanism of cellular uptake. Nanotechnology. 2009;20:425103. 
43. Yuan $Y$, Wang X, Jia G, et al. Pulmonary toxicity and translocation of nanodiamonds in mice. Diam Relat Mater. 2010;19:291-299.

44. Liu T, Li L, Fu C, Liu H, Chen D, Tang F. Pathological mechanisms of liver injury caused by injection of silica nanoparticles. Biomaterials. 2012;33:2399-2407.
45. Nguyen TTB, Chang HC, Wu VWK. Adsorption and hydrolytic activity of lysozyme on diamond nanocrystallites. Diam Relat Mater. 2007; 16:872-876.

46. Barnard AS. Self-assembly in nanodiamond agglutinates. J Mater Chem. 2008;18:4038-4041. 


\section{Supplementary materials Materials and methods}

Red fluorescent nanodiamond (mean size $140 \mathrm{~nm}$ ) was donated by the Institute of Atomic and Molecular Sciences, Academia Sinica, Taiwan. Doxorubicin was purchased from Shenzhen Main Luck Pharmaceuticals Inc (Shenzhen, People's Republic of China). HepG2 cells were provided by the Gene Engineering Center of Shanxi University (Shanxi, People's Republic of China). Dulbecco's Modified Eagle's Medium (DMEM) was purchased from Thermo Fisher Biological and Chemical Product (Beijing, People's Republic of China). Fetal bovine serum was purchased from Hangzhou Sijiqing Biological Engineering Materials Co, Ltd (Hangzhou, People's Republic of China). Trypsin was

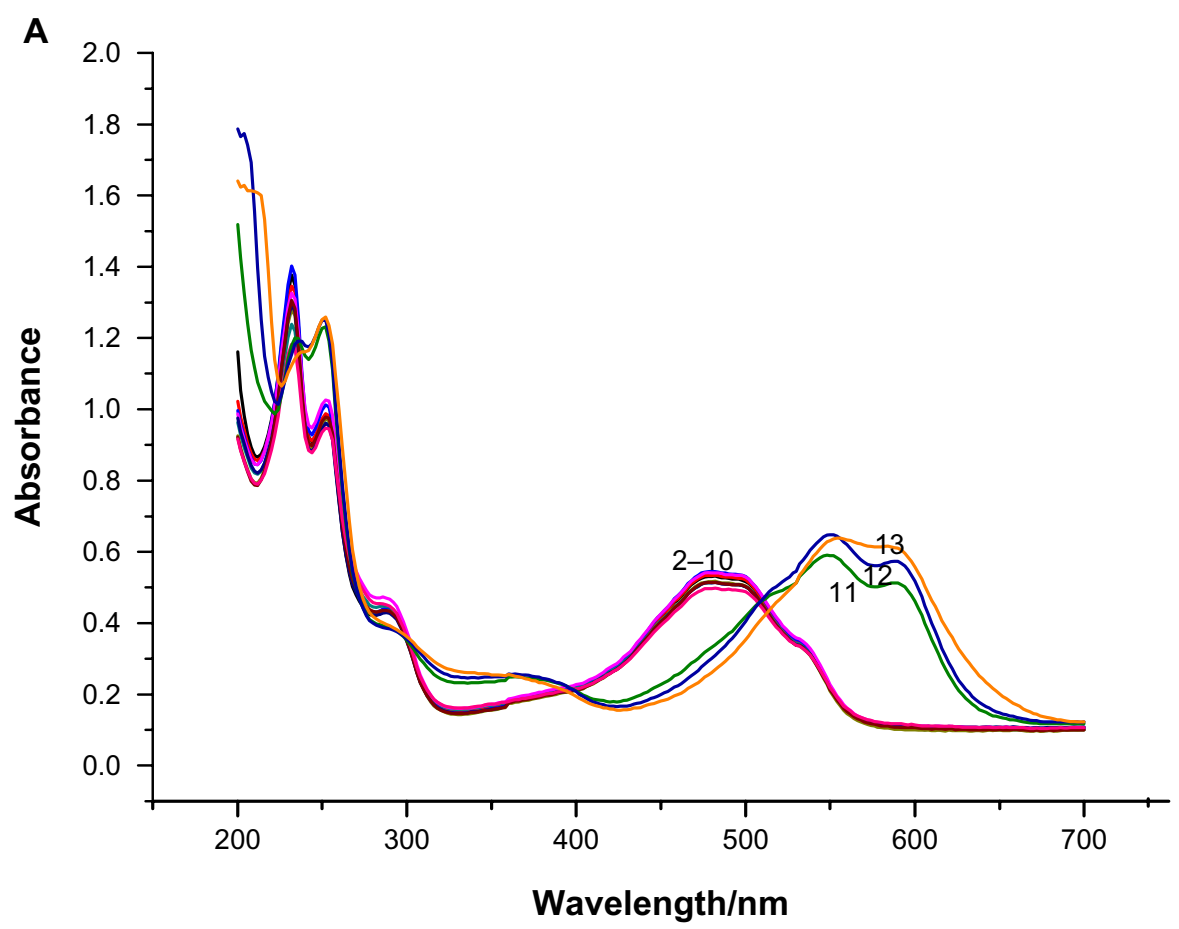

B

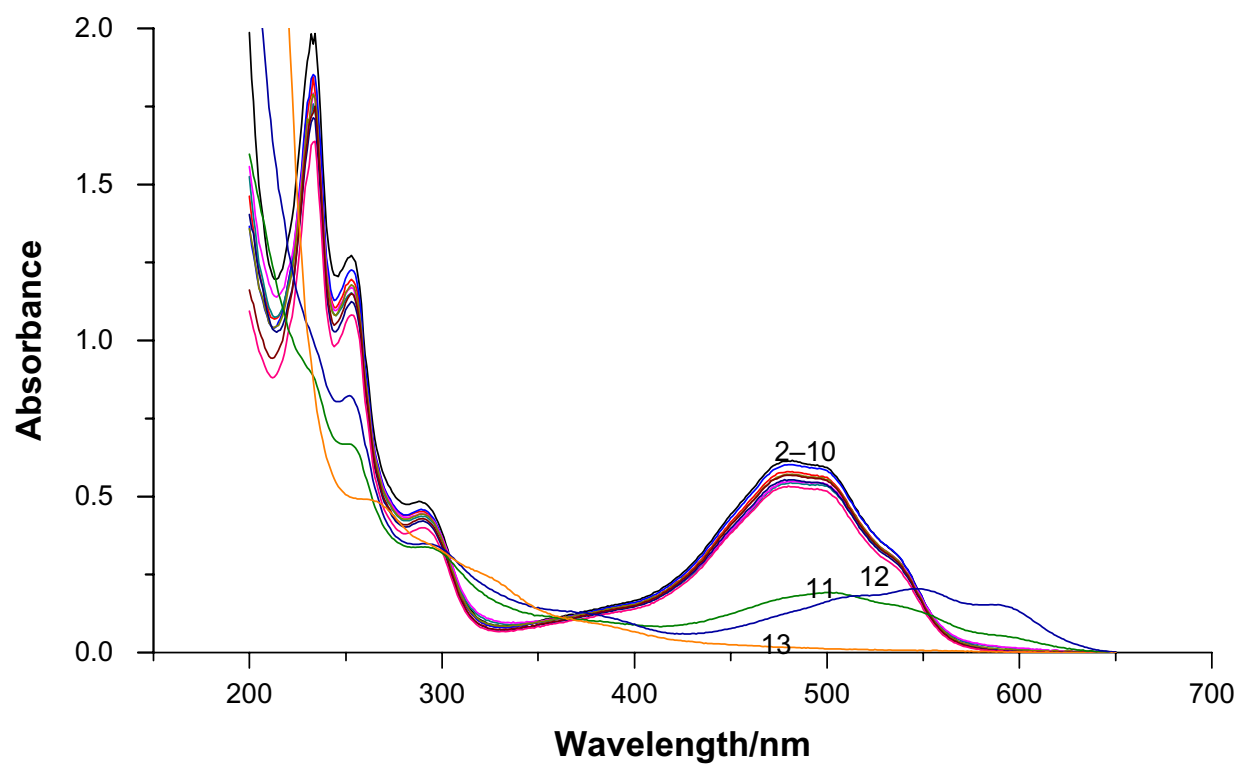

Figure SI (A) Ultraviolet-visible absorption spectra for doxorubicin in different $\mathrm{pH}$ solution (the $\mathrm{pH}$ value represents data without addition of doxorubicin) for testing at different time points. The peak position and peak intensity showed no significant changes in a series of $\mathrm{pH}$ strengths $(2-10)$. However, at $\mathrm{pH} I \mathrm{I}-\mathrm{I}$, the absorption intensity of doxorubicin increased and shifted from $480 \mathrm{~nm}$ to $550 \mathrm{~nm}$. (B) Ultraviolet-visible absorption spectra for doxorubicin in pH solutions of different strength overnight. The peak position and peak intensity showed no significant changes in a series of $\mathrm{pH}$ strengths $(2-10)$ for 17 hours. However, at $\mathrm{pH} \mathrm{II}-\mathrm{I} 3$, the absorption intensity and peak position of doxorubicin changed markedly, indicating that the structure of doxorubicin was destroyed. 


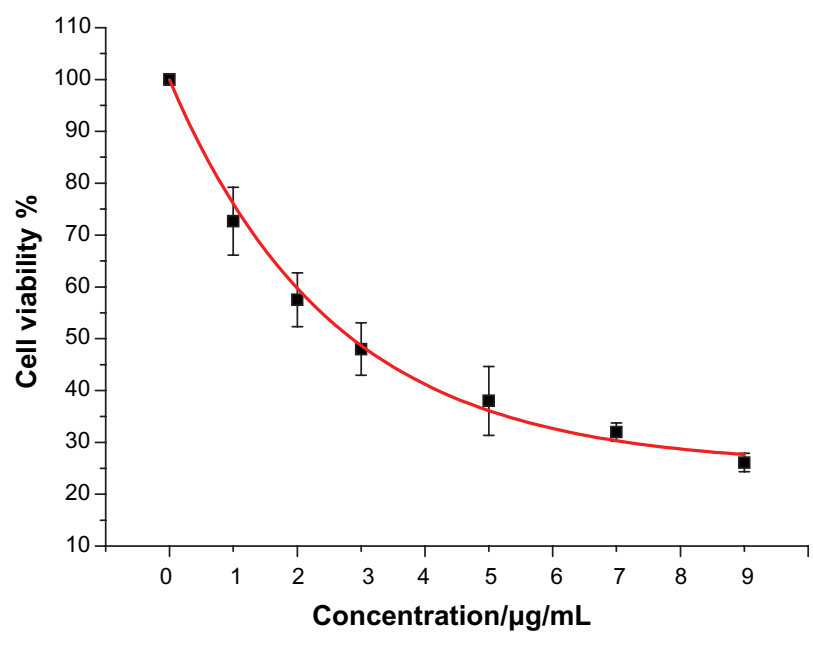

Figure S2 HepG2 cell viability assay by MTT (3-[4,5-dimethylthiazol-2-yl]-2,5 diphenyltetrazolium bromide) with addition of ND-DOX, whereby HepG2 cells were treated for 72 hours. The data are represented as the mean \pm standard deviation $(n=6)$. Abbreviation: ND-DOX, nanodiamond-doxorubicin complex.

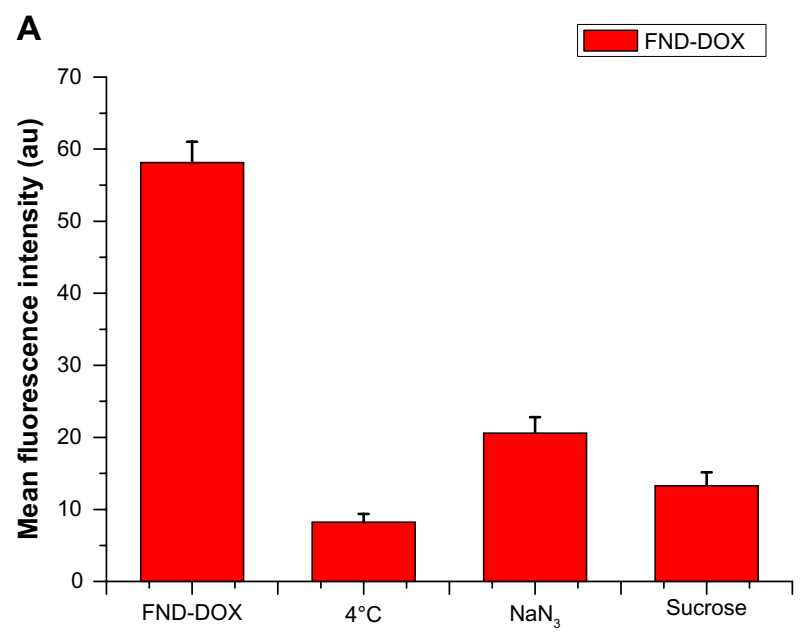

B

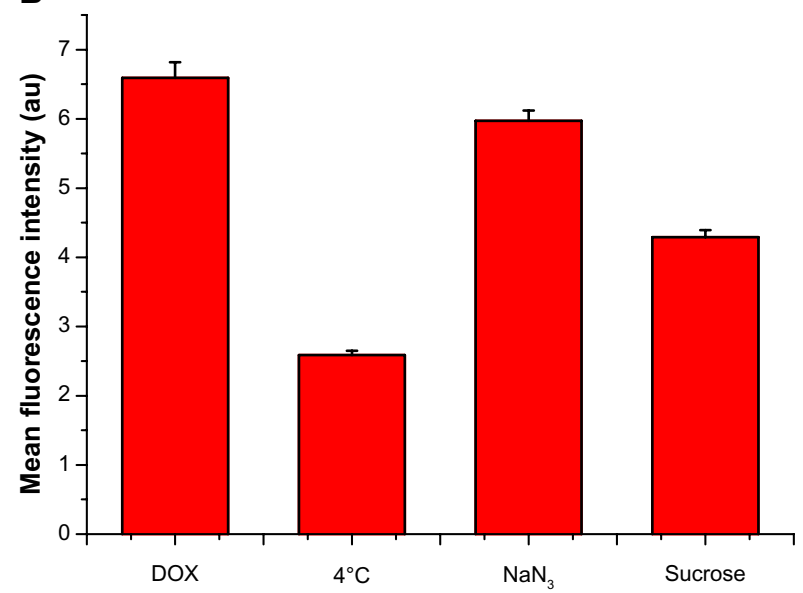

Figure S3 Quantitative analysis of mechanistic study of the uptake of FND-DOX (A) or doxorubicin (B) by HepG2 cells. Variation of the mean fluorescence intensity of internalized FND-DOX or doxorubicin under different treatment conditions: $0.45 \mathrm{M}$ sucrose, $0.07 \mathrm{M} \mathrm{NaN}_{3}$, and $4^{\circ} \mathrm{C}$. The cells were incubated with FND-DOX (86 $\mu \mathrm{g} / \mathrm{mL} F N D+6 \mu \mathrm{g} / \mathrm{mL}$ doxorubicin) or doxorubicin $(6 \mu \mathrm{g} / \mathrm{mL})$.

Abbreviations: ND-DOX, nanodiamond-doxorubicin complex; FND, red fluorescence nanodiamond; DOX, free doxorubicin. purchased from Sino-American Biotechnology Company (Zhengzhou, People's Republic of China). Ethylenediamine tetraacetic acid was obtained from Beijing Solarbio Science and Technology Co, Ltd (Beijing, People's Republic of China). Filtered water (EMD Millipore Corporation, Billerica, MA, USA) was used for all aqueous solutions. The equipment and instruments used are as follows: a water-jacketed $\mathrm{CO}_{2}$ cell incubator (Shanghai Lishen Scientific Instruments Co, Ltd, Shanghai, People's Republic of China), an FQ-100DE numerical control ultrasonic wave washing machine (Kunshan Ultrasonic Instrument Co, Ltd, Kunshan City, People's Republic of China), a full automatic microplate reader (Model 550, Bio-Rad Laboratories Inc, Hercules, CA, USA), a flow cytometer (FACSCalibur ${ }^{\mathrm{TM}}$, BD Biosciences, Franklin Lakes, NJ, USA), and a transmission electron microscope (JEM-1011, JEOL Ltd, Tokyo, Japan).

\section{Effect of $\mathrm{pH}$ on ultraviolet-visible absorption spectra for doxorubicin}

Doxorubicin solutions in a series of different $\mathrm{pH}$ values (2.0-13.0) were prepared using hydrochloric acid and sodium hydroxide solution. Ultraviolet-visible absorption spectra for doxorubicin were recorded using an HP8453 ultravioletvisible spectrophotometer at different time points.

\section{Cell viability assay}

Healthy HepG2 cells $\left(5 \times 10^{3}\right)$ were plated into 96-well microtiter plates (Nunc A/S, Roskilde, Denmark). After 12 hours, the culture medium was replaced by fresh medium $(100 \mu \mathrm{L})$ containing either doxorubicin (final concentrations 1, 2, 3, 5,7 , and $9 \mu \mathrm{g} / \mathrm{mL}$ ) or a nanodiamond-doxorubicin complex (ND-DOX, equivalent of doxorubicin). The cells were then incubated for 72 hours. After incubation, the solutions were aspirated and replaced with DMEM $(180 \mu \mathrm{L})$, followed by addition of $5 \mathrm{mg} / \mathrm{mL}$ MTT (3-[4,5-dimethylthiazol-2-yl]-2,5 diphenyltetrazolium bromide) solution $(20 \mu \mathrm{L}$, final concentration $0.5 \mathrm{mg} / \mathrm{mL}$ ) and incubated for 4 hours. Unreacted dye was removed by aspiration; the insoluble formazan crystals were dissolved by adding dimethylsulfoxide $(200 \mu \mathrm{L})$ to each well, shaken for 10 minutes, and measured spectrophotometrically in an enzyme-linked immunosorbent assay reader at a wavelength of $490 \mathrm{~nm}$. Each group contained six samples. The spectrophotometer was calibrated to zero absorbance using culture medium without cells. The relative cell viability (\%) related to the control groups was calculated as follows:

$$
\text { Cell viability }=\left[\mathrm{A}_{490}(\text { sample }) / \mathrm{A}_{490}(\text { control })\right] \times 100 \%
$$




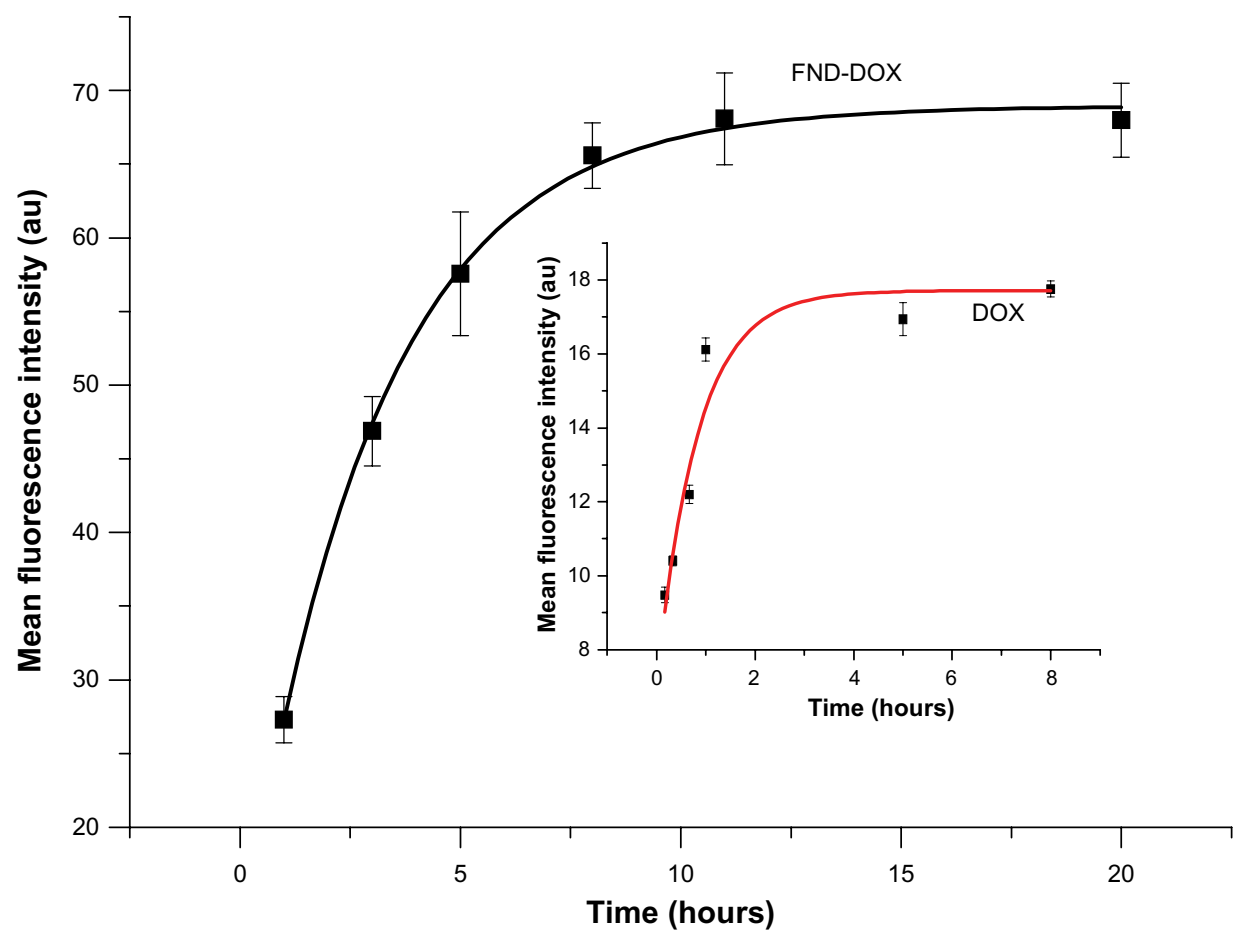

Figure S4 Kinetics of the cellular uptake of FND-DOX ( $86 \mu \mathrm{g} / \mathrm{mL} F N D+6 \mu g / \mathrm{mL}$ doxorubicin). Inset shows the kinetics of cellular uptake of free doxorubicin (6 $\mu g / \mathrm{mL})$. Abbreviations: FND, red fluorescence nanodiamond; DOX, free doxorubicin; h, hour.

A

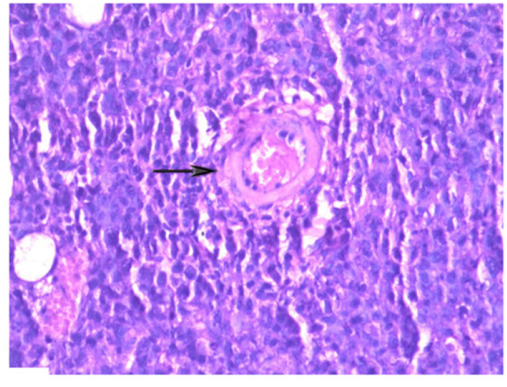

C

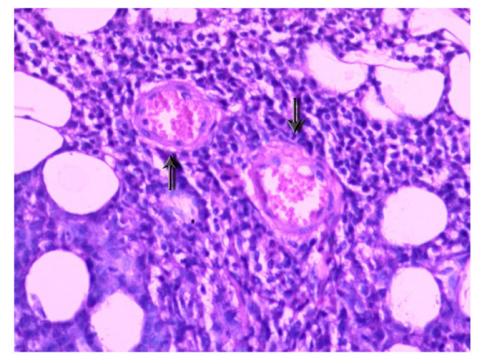

B

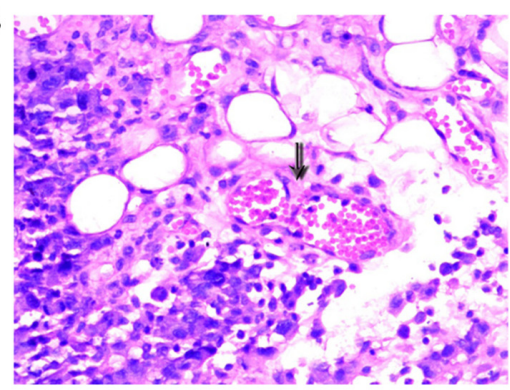

D

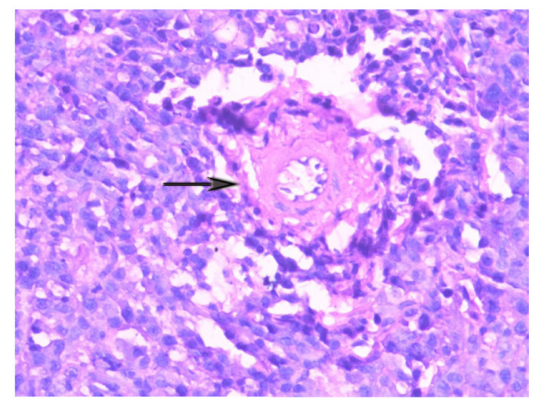

E

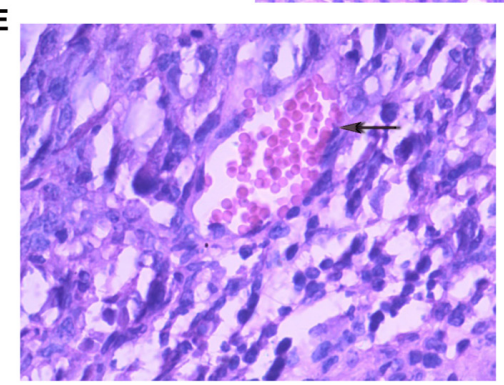

Figure S5 Ex vivo tumor angiogenesis imaging of representative tumor sections in a mice with $\mathrm{H} 22$ liver cancer cells model for (A) control, (B) phosphate-buffered saline, (C) NDs, (D) doxorubicin, and (E) ND-DOX group. Black arrows show the location of tumor angiogenesis. Abbreviations: NDs, nanodiamond particles; ND-DOX, nanodiamond-doxorubicin complex. 
where $\mathrm{A}_{490}$ (sample) is the absorbance value of cells treated with ND-DOX or free doxorubicin, and $\mathrm{A}_{490}$ (control) is the absorbance value of control cells.

\section{Study of cell uptake mechanism}

To determine the cell uptake mechanism for a nanodiamond drug carrier (FND-DOX), flow cytometry analysis was used and four separate experiments were carried out. In the first experiment, the effect of temperature on cell uptake was studied by incubating the cells with FND-DOX or doxorubicin at $4^{\circ} \mathrm{C}$ for 2 hours. In the second experiment, the effect of ATP depletion on cell uptake was assessed. The cells were preincubated in medium supplemented with $0.08 \mathrm{M} \mathrm{NaN}_{3}$ for 15 minutes at $37^{\circ} \mathrm{C}$, followed by incubation for 2 hours. In the third experiment, the influence of a hypertonic solution on uptake was studied. The cells were pretreated with $0.45 \mathrm{M}$ sucrose for 30 minutes at $37^{\circ} \mathrm{C}$ prior to incubation with FND-DOX or doxorubicin. The control experiment was carried out by incubating FND-DOX or doxorubicin in DMEM with $10 \%$ fetal bovine serum at $37^{\circ} \mathrm{C}$. The cell uptake of FND-DOX or doxorubicin was analyzed by flow cytometry. Prior to the flow cytometry analysis, the cells were washed three times with ice-cold phosphate-buffered saline to remove free FND-DOX or doxorubicin and then harvested by trypsinization.

\section{Kinetics of cell uptake for ND-DOX and doxorubicin}

HepG2 cells were cultured in DMEM with $10 \%$ fetal bovine serum at $37^{\circ} \mathrm{C}$ in a humidified atmosphere with $5 \% \mathrm{CO}_{2}$. To study whether the cell uptake of FND-DOX or doxorubicin is time-dependent, HepG2 cells were seeded in $35 \mathrm{~mm}$ dishes $\left(\sim 2 \times 10^{5}\right.$ cells per dish) in DMEM with $10 \%$ fetal bovine serum. After incubation for 18 hours, the culture medium was replaced by the same medium containing FND-DOX or doxorubicin for both time dependence measurements. The cell uptake of FND-DOX or doxorubicin was determined by flow cytometry. Prior to the flow cytometry analysis, the cells were washed three times with ice-cold phosphate-buffered saline to remove free FND-DOX or doxorubicin and then harvested by trypsinization.

\section{Statistical analysis}

Data were analyzed for significance by the Student's $t$-test. The results were considered statistically significant at a $P$-value $<0.05$.
International Journal of Nanomedicine

\section{Publish your work in this journal}

The International Journal of Nanomedicine is an international, peerreviewed journal focusing on the application of nanotechnology in diagnostics, therapeutics, and drug delivery systems throughout the biomedical field. This journal is indexed on PubMed Central, MedLine, CAS, SciSearch ${ }^{\circledR}$, Current Contents ${ }^{\circledR} /$ Clinical Medicine,

\section{Dovepress}

Journal Citation Reports/Science Edition, EMBase, Scopus and the Elsevier Bibliographic databases. The manuscript management system is completely online and includes a very quick and fair peer-review system, which is all easy to use. Visit http://www.dovepress.com/ testimonials.php to read real quotes from published authors. 